\title{
Design of a Predictive Scheduling System to Improve Assisted Living Services for Elders
}

\author{
VALERIA SOTO-MENDOZA, CICESE Research Center, Mexico \\ J. ANTONIO GARCÍA-MACÍAS, CICESE Research Center, Mexico \\ EDGAR CHÁVEZ, CICESE Research Center, Mexico \\ ANA I. MARTINEZ-GARCÍA, CICESE Research Center, Mexico \\ JESÚS FAVELA, CICESE Research Center, Mexico \\ PATRICIA SERRANO-ALVARADO, Université de Nantes, LINA Lab, France \\ MAYTHÉ R. ZÚÑIGA ROJAS, CICESE Research Center, Mexico
}

\begin{abstract}
As the number of older adults increases, and with it the demand for dedicated care, geriatric residences face a shortage of caregivers, who themselves experience work overload, stress and burden. We conducted a long-term field study in three geriatric residences to understand the work conditions of caregivers with the aim of developing technologies to assist them in their work and help them deal with their burden. From this study we obtained relevant requirements and insights of design that were used to design, implement and evaluate two prototypes for supporting caregivers' tasks (e.g. electronic recording and automatic notifications), in order to validate the feasibility of their implementation in-situ and the technical requirements. The evaluation in-situ of the prototypes was conducted for a period of four weeks. The results of the evaluation, together with the data collected from six months of use, motivated the design of a predictive schedule. Such design was iteratively improved and evaluated in participative sessions with caregivers. PRESENCE, the predictive schedule we propose, triggers real-time alerts of risky situations (e.g. falls, entering off-limits areas such as the infirmary or the kitchen) and, informs caregivers of routine tasks that need to be performed (e.g. medication administration, diaper change, etc.). Moreover, PRESENCE helps caregivers to record caring tasks (such as diaper changes or medication) and wellbeing assessments (such as the mood), which are difficult to automatize. This facilitates caregiver's shift handover, and can help to train new caregivers by suggesting routine tasks and by sending reminders and timely information about the residents. It can be seen as a tool to reduce the workload of caregivers and medical staff. Instead of trying to substitute the caregiver with an automatic caring system, as proposed by others, we propose the design of our predictive schedule system that blends caregiver's assessments and measurements from sensors. We show the feasibility of predicting caregiver's tasks and a formative evaluation with caregivers that provides preliminary evidence of its utility.
\end{abstract}

Categories and Subject Descriptors: J.3 [Life and Medical Sciences]: Health

General Terms: Design, Human Factors, Measurement

Additional Key Words and Phrases: Assistive living systems, Activities of daily living (ADL), Elderly care

\section{ACM Reference Format:}

Valeria Soto-Mendoza, J. Antonio García-Macías, Edgar Chávez, Ana I. Martínez-García, Jesús Favela, Patricia Serrano-Alvarado, and Maythé R. Zúñiga Rojas, 2013. Predictive Scheduling to Improve Assisted Living Services for Elders. ACM Transactions on Intelligent Systems and Technology (ACM TIST). 9, 4, Article 39 (March 2010), 22 pages.

DOI:http://dx.doi.org/10.1145/0000000.0000000

This work is supported by the Mexican National Council for Science and Technology (CONACyT).

Author's addresses: V. Soto-Mendoza, J. Antonio García-Macías, Edgar Chávez, Ana I. Martinez-García, Jesús Favela, and Maythé R. Zúñiga Rojas, Computer Science Department, CICESE Research Center, Baja California, México; Patricia Serrano-Alvarado, Université de Nantes, Lina Lab, France.

Permission to make digital or hardcopies of part or all of this work for personal or classroom use is granted without fee provided that copies are not made or distributed for profit or commercial advantage and that copies show this notice on the first page or initial screen of a display along with the full citation. Copyrights for components of this work owned by others than ACM must be honored. Abstracting with credits permitted. To copy otherwise, to republish, to post on servers, to redistribute to lists, or to use any component of this work in other works requires prior specific permission and/or a fee. Permissions may be requested from Publications Dept., ACM, Inc., 2 Penn Plaza, Suite 701, New York, NY 10121-0701 USA, fax +1 (212) 869-0481, or permissions@acm.org.

(C) 2010 ACM 1539-9087/2010/03-ART39 $\$ 15.00$

DOI:http://dx.doi.org/10.1145/0000000.0000000 


\section{INTRODUCTION}

The world's population is aging and, as a consequence, the number of older adults has experienced a sharp increase in recent years, increasing with it the demand for dedicated care (World Health Organization 2012). The elders, by their own, or via their closest relatives decide to move to geriatric centers, also called residences or nursing homes (Hossain 2014), which are places designed according to the needs of older adults (residents) with specialized personnel to take care of them (Silva et al. 2013).

Despite the specialized services offered, the capacity of geriatric care centers is limited (Bardram 2008), and very often residents greatly outnumber caregivers attending capacity (Fennell et al. 2010). The staff is constantly under work pressure and stress (Yedidia \& Tiedemann 2008) due to emotional wear, rotating shifts, the need for specialized training (Rosenberg et al. 2009) to treat and attend each resident, etc. Moreover, caregivers are required to record the activities performed by the residents of the nursing home. For this, they rely on conventional tools, such as pen and paper (Archer et al. 2011); frequently these records are not up-to-date due to heavy workloads (Blumenthal \& Tavenner 2010). As a consequence all the staff (caregivers, doctors, nurses, etc.) act upon incomplete information (UNFPA 2012). As personnel rotation occurs, newcomers rely on more experienced members in order to get valuable information from them to better perform their jobs. Thus, there is a lot of implicit knowledge in the care of elders, the methods to capture such knowledge are unreliable and error-prone, and knowledge transmission is not systematic.

No attempt has been made, so far, to incorporate valuable information from caregivers into assistive technology to support elders' care processes in residences. This is not an easy task. For a perfect understanding of the care process and, the elders and staff needs, it is necessary to propose an appropriate technology. The grand view of this work is to extract and systematize the essential knowledge behind caring elders, to learn the methods and techniques used by caregivers, and to detect weaknesses and improvement opportunities in nursing homes. Therefore, we conducted a field study to develop suitable assistive applications based on the principal characteristics found in naturalistic environments. This paper discusses the process guiding the design of ad-hoc applications developed to assist elders' without losing sight from caregivers' burden and assessments. The result of our study is the design of a predictive schedule that will help caregivers in their daily activities to diminish burden, as well as a tool to transmit information during shifts in a discreet, transparent and orderly manner.

Our principal finding is the need for an assistive technology able to present a holistic view of the care needs of each resident, without consuming the ever scarce time of the caregivers. Moreover, due to the routine nature of caring, the adequate tool would be a predictive schedule. That is, a regular schedule with some inference mechanisms to suggest tasks, receive timely reminders, monitor elders' location passively, trigger alerts and alarms from risky situations, and a few other abilities like electronic logging and reports.

The above design was possible after a long-term study, which took us to the design and implementation of two prototypes, their corresponding evaluation and several rounds of participative design sessions. All these was done to explore and validate, 
with caregivers, medical staff and in-situ conditions, the main characteristics established for the design of the predictive schedule such as: the informing through notifications about risky situations (SMAMI) and, the electronic recording of annotations from caregivers (SSAMI). Also, as part of the subsequent participative design sessions users suggested important enhancements to consider, which lead to more requirements in the design of this new assistive tool for caregivers in geriatric centers. This paper discusses the process guiding the design, the insights acquired, and how they were incorporated in successive iterations of the predictive schedule named PRESENCE.

The rest of this paper is organized as follows. Section 2 discusses related work. Section 3 presents our research methodology. Section 4, shows the iterative design process that guides the implementation of two functional prototypes. In section 5 we present the design of PRESENCE as a result after the in-situ evaluation. Section 6 presents a discussion of our results and we conclude in Section 7 with some remarks.

\section{RELATED WORK}

In recent years, many assistive technologies have been proposed to assist elders and caregivers to: advise about their nutritional habits (Lázaro et al. 2010), monitor risky behaviors (Kaluža et al. 2011), measure muscle strength with videogames (Zavala-Ibarra \& Favela 2012), socialize and exercise (Cornejo et al. 2012), track activities (BeClose 2013). Others are focused on the improvement of the care process by providing: electronic health records (Kim \& Kim 2010), remote medical attention (Havasi \& Kiss 2008), and intelligent vital signs monitor (Megalingam et al. 2012). Among these assistive technologies, there are Ambient Assisted Living (AAL) systems (Sun et al. 2010) that analyze different data sources from sensors to generate timely information useful for both older adults and the staff working in geriatric centers. This data can be analyzed to identify individual and group patterns from which it can be inferred situations requiring the attention of the caregiver (Fatima et al. 2013). That is, it is possible to generate additional knowledge about the resident from the data collected and sensed (Fayyad et al. 1996). Also, deviations in the behavior of the elderly have been analyzed to detect health risks (Botia et al. 2012) and situations that may require attention (Shin et al. 2011).

Using sensors, it is possible to infer elders' health, by monitoring the level of physical activity performed (Kaluža et al. 2012), as an indicator of their frailty (Bravata et al. 2007). Monitoring and assessing the performance of daily activities is relevant for the continuous assessment of elders' health and independence (Onyimadu et al. 2011). Promising results have been obtained with the combination of sensor and medical data using diverse techniques to determine the level of wellbeing in older adults while performing their daily activities (Suryadevara et al. 2012).

Virtual caregivers have also been proposed to assist the human caregiver in taking basic decisions, asking for human intervention when necessary (Hossain \& Ahmed 2012). In most of the above-mentioned work, the analysis of heterogeneous sensor sources is used to make inferences. However, based on the classification of digital sources proposed in (Zhang et al. 2012), sensor data are only part of the static sensing infrastructure, and it focused on inferring a single type of behavior, e.g. 
mobility patterns. The challenge is in including more context data sources and combining them to improve inferences. In (Rodríguez et al. 2014) an information fusion approach was proposed as a multi-agent system, which manages data from sensors and processes them to infer the activities of residents. The focus of this last work is in presenting statistics from location sensors, and based on the statistics the authors suggest the creation of patterns. Statistics are valuable information to make decisions but, they are not the best form to present and visualize information for fast decision making by caregivers (Skiba 2014).

Another alternative to provide care to older adults is with robotic caregivers (Borenstein \& Pearson 2010)(Sorell \& Draper 2014). Certainly, robotic caregivers are an approach intended to substitute human caregivers (Parks 2010), to encourage elder's cognitive skills (Coeckelbergh 2010), or to avoid isolation as a partner of an elder (Sparrow \& Sparrow 2006). Although there are advantages on the robotic caregivers there are still some elements that limit their adoption as a viable approach for caring older adults (Decker 2008)(Vallor 2011)(Misselhorn et al. 2013). Moreover, robotic caregivers surely represent an option to reduce or even eliminate burden on human caregivers, even though human caregivers will not be replaced by robots in the near future. Our approach considers using caregivers' observations as an input to a prediction model and combines them with data from sensors to provide predictions based on mixed context information. On the other hand, the assistive technology aims to reduce caregivers' burden through simplifying the care processes and tasks (Silva et al. 2013)(Aloulou et al. 2013) (Piau et al. 2014). In this way, technology helps to increase the quality and efficiency of care (Lexis et al. 2013) of older adults. We believe that the predictive schedule would be another mechanism that will help to diminish the caregivers' burden and stress, by the anticipation in registering elders' activities. The time saved enables caregivers to program other kinds of activities with elders (such as, games, chats, hand crafts, etc.) which will impact elders' mood and wellbeing (Shankar et al. 2014).

\section{RESEARCH METHODOLOGY}

The methodology used in this work was iterative and user-centered (Norman \& Draper 1986), and it describes the steps we followed to design the predictive schedule. The methodology consists of the following five phases (see Fig. 1); each one is explained in detail next and developed in the following sections:

1. Initial context comprehension. This step includes acquiring empirical knowledge from the application field, to understand and establish roles, artifacts, tasks, activities, and processes. Qualitative techniques were used such as structured and semi-structured interviews, non-participative observations, shadowing, and process engineering. The above techniques enabled empirical data collection for later analysis and to construct valid theory from qualitative data.

2. Context data analysis. The data acquired in phase 1 were analyzed. All the interviews, observations and annotations were transcribed to execute an iterative analysis seeking for concepts and categories. This process enabled us to identify insights, needs and requirements within the specific context to develop prototypes to explore the use of technology for electronic recording of activities and, for sending notifications about risky situations 
automatic detected, in order to present a novel and complete design of an assistive tool for caregivers in geriatric centers, the predictive schedule.

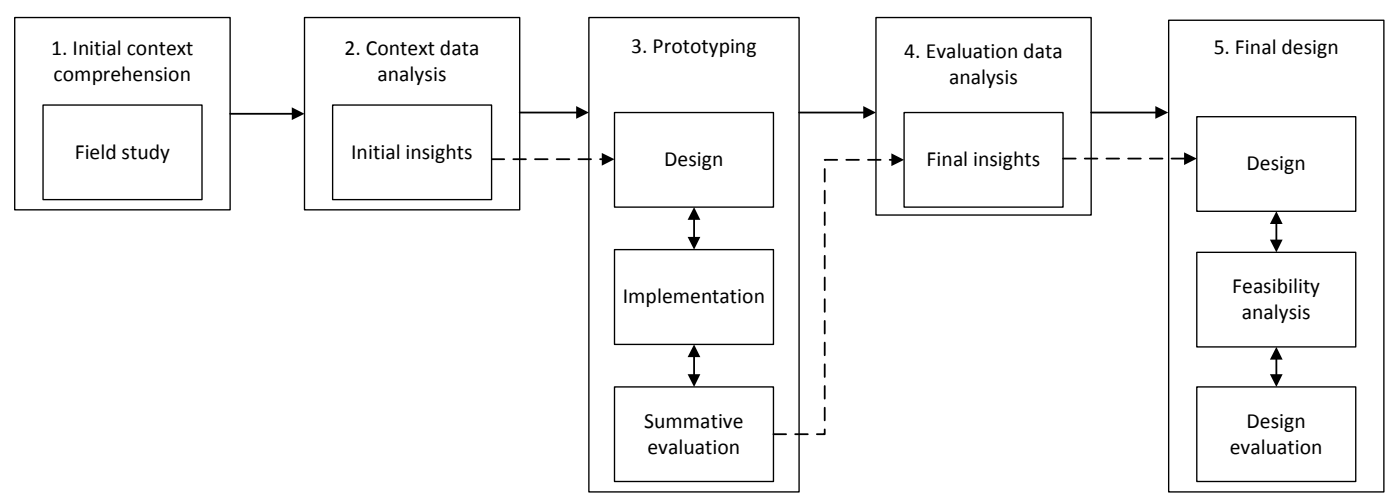

Fig. 1. Research methodology.

3. Prototyping. In this phase, the relevant elements from the previous phase were incorporated in two prototypes. The elements we embedded and tested with the prototypes were the electronic registering of activities (SSAMI) and the informing through notifications about risky situations and sensor readings (SMAMI). Both prototypes, SSAMI and SMAMI, were implemented and evaluated based on the insights defined formerly to improve the care of residents and the awareness of the caregivers about the residents. The implementation included the development of tools and applications around the prototypes. Finally, the prototypes were evaluated in-situ to validate our first elements with reliable information and, to add value to the design of the predictive schedule with some unpredicted and real situations arisen in the "wild".

4. Evaluation data analysis. The data obtained from the evaluation of the prototypes were analyzed and incorporated into the design of the predictive schedule. The perceptions and technicalities about the users of electronic registering (with SSAMI) and automatic notifications of risky situations (with SMAMI) were studied to find gaps, which were considered to get the final design insights of the predictive schedule.

5. Final design. The system's main requirements were validated with the insitu evaluation of the prototypes, and more final insights were obtained for designing the predictive scheduling system. We also explored the viability of the predictive schedule by conducting a participative design session with caregivers and medical staff. We present the analysis of the main results that gave us evidence about the utility and viability of implementation of the predictive schedule for caregivers.

\section{DESIGN PROCESS}

The following sections detail the methodology we used to collect, analyze and process data from personnel and residents of the geriatric residences. With a nonparticipatory observation (Section 4.1) we determined the features to be monitored (Section 4.2), and from that we designed and developed prototypes (Section 4.3). 


\subsection{Initial context comprehension}

We conducted a field study to investigate the caring needs of residents of geriatric centers, and to learn the methods and procedures used for the care of older adults. The field study took place in three different institutions in the state of Baja California in the northwest of Mexico, two of them private and one public. We conducted a non-participatory observation (180 hours), and a total of 19 semi structured interviews with caregivers and residents of the three institutions. Residents came from different socioeconomic strata; had different levels of support from their social network and also variable levels of health and physical and mental fitness. This implies a variety of specialized care needs to be met on an individual basis. From the data gathered, we performed an analysis to obtain a classification of the main activities of both the residents and the caregivers. This analysis included a thorough and comprehensive review of the observation logs and interviews using methodologies such as grounded theory (Glaser \& Strauss 2012) and sequential analysis (Wald 2004), and process engineering (Davenport 1993) covering quantitative and qualitative perspectives.

We transcribed manually 19 semi-structured interviews conducted in the different residences. Using some techniques of grounded theory (open and axial coding) we found the main activities performed by caregivers and residents. Then the activities were classified according to the Activities of Daily Living (ADL) classification and their relevance for caregivers, and we formed groups of activities and tagged them. Next we present the definition and classification of these activities.

- Information management. Activities related to the collection and consultation of relevant information to the process of elders' care. It covers filling and updating reports hourly by hand, one by each shift, and reporting eventualities, if any. It includes accessing information from the medical record, filling in nursing sheets, and monitoring and registering information about meals, excretions, urination and eventualities in the notebooks.

- Coordination. Activities related to staff management, e.g. the meetings for planning and assigning work tasks, planning special diets, inventory control, operational meetings, collecting and verifying medications for the elders, talks between the staff and reviews about general aspects.

- Care. Activities related to maintaining the wellbeing of the elders. Care activities are divided into:

- Medical attention. Taking vital signs, medication intake, medical consultations and visits, and preparing medications or some medical devices in the nursing station.

- Hygiene. Bathing, assisting in the bathroom, changing clothes, changing diapers and grooming (haircut, cutting nails, brushing teeth, washing the face, etc.)

- Recreation. Walking in the garden, watching movies or the T.V., playing games in the living room, listening to music and going out of the residence with family or friends.

- Feeding. Feeding or providing food to the elders.

- Service. Getting elders out of bed, talking with the elders, checking elders' status, awakening, and responding bell calls.

- Rest. Residents resting in their room or sleeping. 
- Support. The support activities are those additional activities supporting the maintenance, cleanliness and order of the residence. Washing, drying and folding laundry; ordering things in the kitchen or cupboard; preparing meals; receiving family and visitors; maintenance of the facilities; residence cleaning; answering the phone; and opening or closing the door of the residence.

- Personal time. It covers personal and informal chats between caregivers or staff, checking their mobile phone, time for eating, and taking personal calls.

Once, identified the main activities, we proceeded to model the care process. The methodology we used was process engineering (Curtis et al. 1992) from which we built a rich picture (Checkland 1989) with the general view of the process. A rich picture gives a comprehensive and useful view of the process it models. This picture (see Fig. 2) conceptualizes a general view of the process, its inputs, outputs, roles and the relationships among them. For instance, the caregivers change clothes of the resident (process), s/he needs the clothes (inputs) and contact with the resident (relationships) to put them on. With the above procedure we obtained qualitative, valuable information for the managers of the residence. We also transcribed the 180 hours of observations gathered and performed a quantitative analysis using sequential analysis of the activities. The analysis of all these results led to the design of the assistive technology for the residents and caregivers as describe in the next sections.

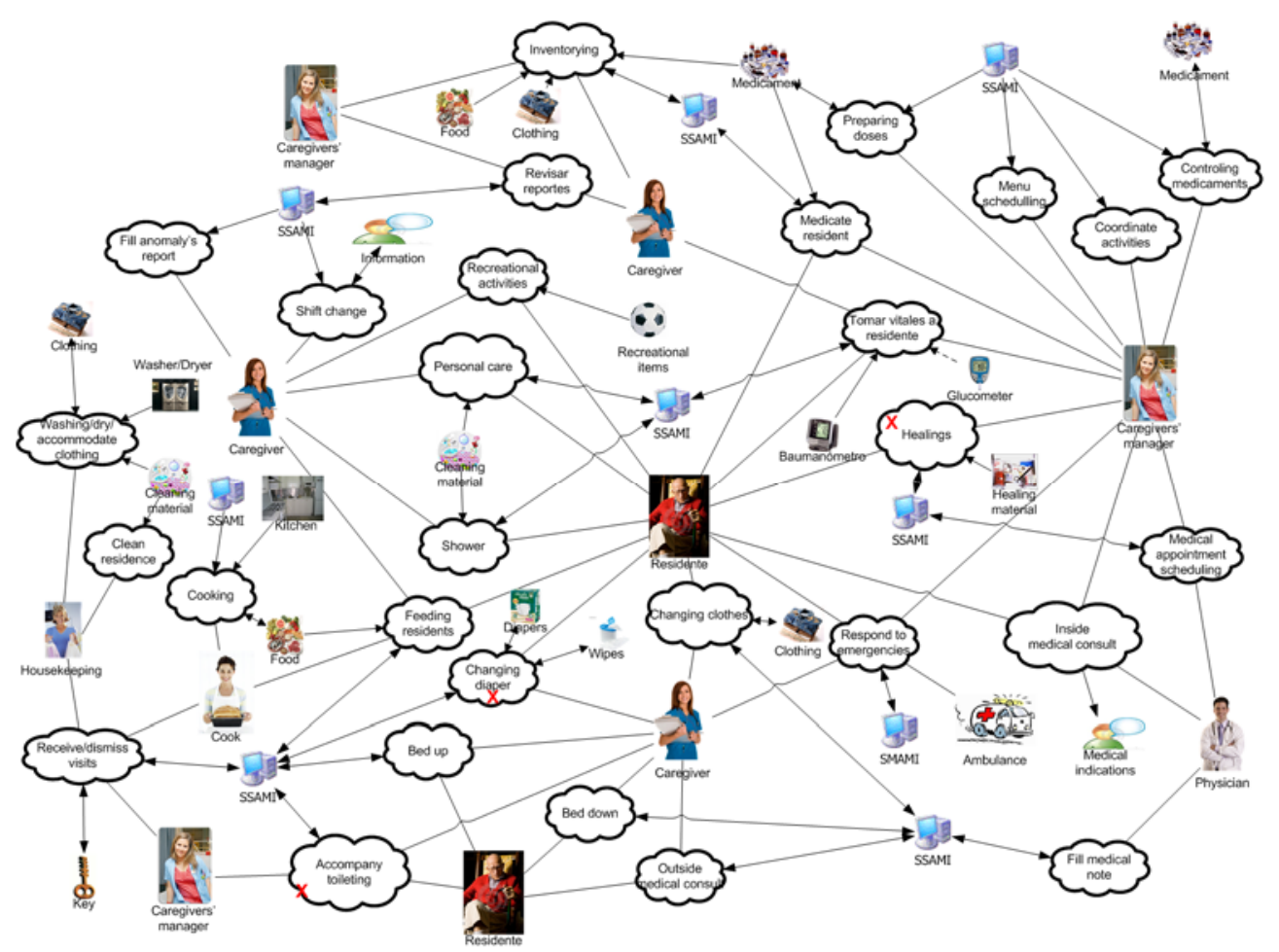

Fig. 2. Rich picture of the care process followed in the geriatric centers studied. 


\subsection{Data analysis}

From the data analysis a list of design insights, technical requirements, and their rationale, were obtained as follows.

Insight \#1. It is important to provide awareness to the caregiver about pending tasks and risky situations, without interrupting the current task.

Technical requirement \#1.1. The user interface and the mode of interaction of the system should compromise between alerting and distracting. For example, alerts should be easily lifted, without disappearing from the roster.

Technical requirement \#1.2. The system should detect when the caregiver is busy.

In the field study, we found that more than one third of the caregivers' time is dedicated directly to the resident's care (37\%). The time a caregiver spends with a resident depends on his/her general health and physical condition. While some of them can be very independent, others may be in need of more care and attention due to its medical conditions (e.g. Alzheimer, Parkinson). We observed that sometimes caregivers spent a considerable amount of time with a single resident, letting others waiting for attention; hence it is essential to find new ways to increase direct contact with all the residents. The numbers above the bars in Fig. 3a indicate the number of times that caregivers perform each type of activity, and Fig. $3 \mathrm{~b}$ shows the number of hours that caregivers dedicate to a specific activity (as defined above in 4.1) and the percentage of the total time that each activity represents.

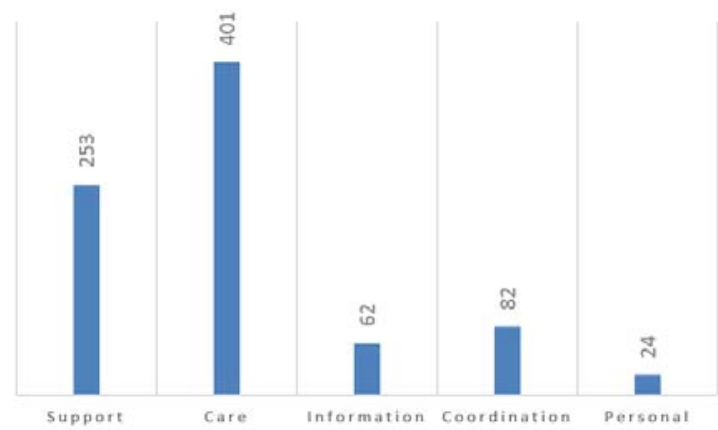

a)

\begin{tabular}{|r|c|c|}
\cline { 2 - 3 } \multicolumn{1}{c|}{} & $\begin{array}{c}\text { Time } \\
\text { (in hours) }\end{array}$ & Percentage \\
\hline Support & $21: 43$ & $37 \%$ \\
\hline Care & $21: 26$ & $37 \%$ \\
\hline Information & $6: 52$ & $12 \%$ \\
\hline Coordination & $4: 06$ & $7 \%$ \\
\hline Personal & $4: 29$ & $8 \%$ \\
\hline
\end{tabular}

b)

Fig. 3. Frequency, total time and percentage of activities considering the time of the caregivers.

Insight \#2. Caregivers must know at all times in which area of the residence elders are and what they are doing.

Technical requirement \#2.1. A real-time indoors location module should be included to determine each elder whereabouts. 
Technical requirement \#2.2. A real-time activity recognition mechanism should be included to know what the elders are doing.

Data analysis revealed that caregivers spend an arbitrary amount of time with each resident, depending on the level of care required. Since the number of caregivers is limited the above implies loosing direct contact with residents under their responsibility.

Insight \#3. It is necessary to provide a signal of alert for a busy caregiver within seconds of a risky situation to prevent a compromise to the elders' health.

Technical requirement \#3.1. An alarm signal should be sent to all the staff when an elder is detected in a risky situation to maximize the chances of timely attention to the elder's condition.

We found that some activities of the residents were not perceived by the caregivers. For example, it is almost impossible to follow all the time an elder who is wandering without assigning one dedicated caregiver to this task. This is particularly sensitive if the caregiver does not notice when a resident enters an off limits or dangerous area such as the kitchen or the infirmary. Moreover an elder might fall without a caregiver noticing, which is risky.

Insight \#4. Due to economic restrictions, care centers will most likely be understaffed. Therefore, there is a need to provide tools to support this situation, in particular concerning the care needs.

Technical requirement \#4.1. The design should plan for understaffing. Care time should be administered and prioritized by care needs.

Due to the diversity of activities that caregivers perform within the residence, on some days the workload exceeds their response capabilities. This situation can be seen as an understaffing problem; nevertheless, even in the hypothetical case of having one caregiver per resident it is possible that at some moment (perhaps for a few seconds), the caregiver can lose eye contact with the resident and a fall might occur. Hiring more personnel can be a solution; however it would increase the costs of care. Hence, there exists a tradeoff between those alternatives. In our study we found that caregivers perform all sort of activities, for instance, from our case study, Fig. 4 shows the workload of two caregivers on two separate days. In one of them (Monday) the absence of kitchen staff compromised care activities when preparing lunch.

Insight \#5. A planning tool and means to measure the degree of completion of planned tasks should be incorporated. The residents in geriatric institutions have routines and the staff plans on this routines, which be carried out.

Technical requirement \#5.1. A module to detect repetitive patterns and a planning tool need to be included in the design. It also should include a tool to measure the degree of adherence to the scheduled program. 
During the time we observed residents and caregivers within the residence, we could establish that their activities have low variability or, complementarily, high repeatability. Moreover, caregivers and residents followed a repetitive program of activities, i.e. every day the residents followed the same set of activities and they rarely varied that routine. This characteristic of periodicity enables, in principle, detecting when residents deviate from their usual routine.

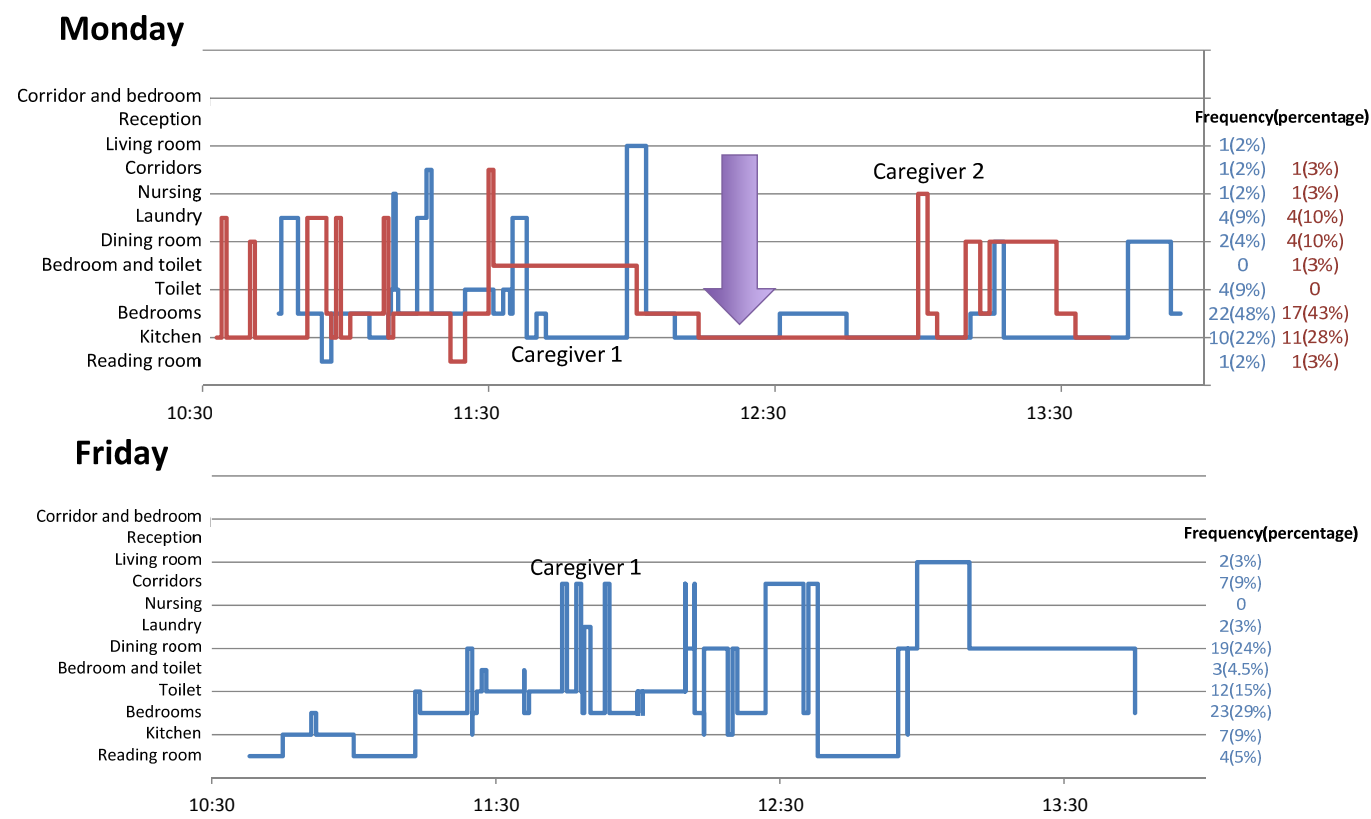

Note: Caregiver 1 represented by the blue line and Caregiver 2 represented by the red line. In Friday, Caregiver 2 was outside of the residence.

Fig. 4. Workload distribution of the caregivers.

Insight \#6. A mechanism to exploit the correlation between the time and locations where activities are being performed is needed, together with the inference of activities given time and location.

Technical requirement \#6.1. The system should include an inference module to predict the activity, given the location of the elder and the time of the day.

We also noticed that the activities of the residents have a high correlation with their location. In some cases, location and time of the day are enough to infer the activity. For example, changing clothes (within care activities) of a resident at morning is usually performed inside the bedroom (see Fig. 5).

Insight \#7. It is necessary to automate the activity records, at the present they are in paper and are redundant.

Technical requirement \#7.1. Daily activities should be logged using an electronic system capable of performing historical searches. 
Information management consisted in recording by hand all the activities residents did during the day. The caregivers must write on paper hour by hour the recent activity of each resident. They had an hour-by-hour register and a notebook of anomalies. At the end of each shift, caregivers should send a report with the same information written on the register. Sometimes the caregivers spent a few minutes after the end of the shift just to complete or correct the records. No electronic record was implemented before, all the records were written in paper and the same information had to be filled in, in up to three different formats. It is clearly a waste of time to invest valuable resources in this kind of repetitive task.

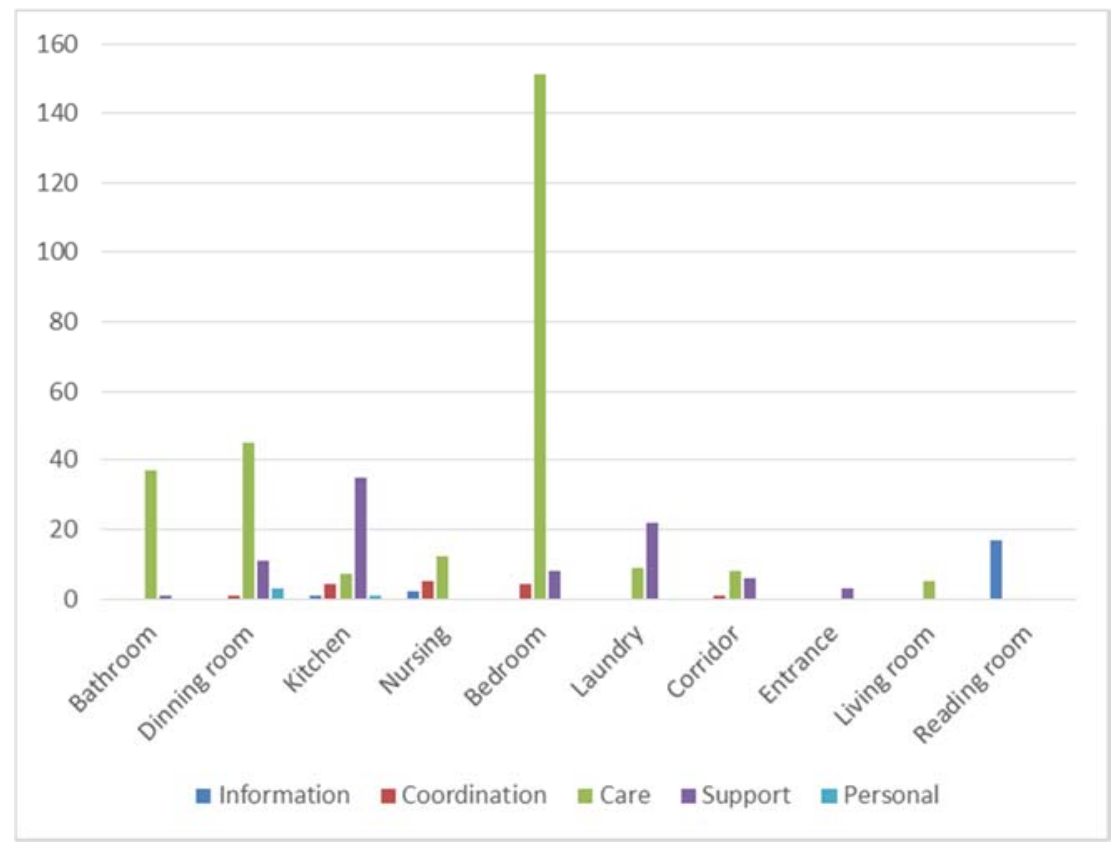

Fig. 5. Frequent places where activities are performed.

Insight \#8. The privacy of residents and their data are principal concerns that should be taken into consideration.

Technical requirement \#8.1. A module managing privacy with hierarchical methods, user profiles and clearance levels, formally proofed to protect the elders' data should be included.

We observed in the residence that the caregivers and staff were responsible for the information of the residents. The control of information was performed manually and the records were available to all staff members and whoever entered the area. The residents and family signed a consent agreement about the residents' information and care. The residence has the responsibility over both the data and the wellbeing of each resident; the storage and management of information should be done in a reliable, safe and systematic way.

The previous analysis provided us with relevant insights of design to be considered for designing supportive technologies for caregivers in geriatric centers: for electronic registering of elders and informing caregivers through notifications about risky situations related with them. In the next section we validate these 
characteristics with the development of two prototypes that cover the requirements established with the insights captured in-situ.

\subsection{Prototyping}

As just mentioned, after the field study, we validated the preliminary design characteristics established for the predictive schedule, by building two prototype systems based on the extracted technical requirements. The design decisions taken during the development and implementation of the prototypes were incorporated into the final design insights and technical requirements of the predictive schedule, to solve the problems stated in the previous phase of the methodology. Both prototypes were designed to have a small cognitive load, and a friendly interface to avoid overwhelming caregivers (Insight \#1, Technical requirement \#1.1) because usually the number of residents exceeded the capabilities of the staff in elderly care centers. Moreover, the use of electronic records could reduce the time of information management (Kim \& Kim 2010)(Su \& Yude 2012); as a consequence, the time remaining could be redirected to increase the quality of the care.

We developed two prototype applications: one for electronic recording of elders' activities (SSAMI - Sistema de Seguimiento de Adultos Mayores Institucionalizados, spanish acronym for Journaling System for Residents) and the second for informing caregivers through notifications of risky situations related with elders (SMAMI Sistema de Monitoreo de Adultos Mayores Institucionalizados, spanish acronym for Monitoring System for Residents). They were designed to run on Android smartphones that could be carried for caregivers at all time, and in all places in the residence. The prototypes are described below.

\subsubsection{Prototype 1. SSAMI.}

Purpose.

The aim of this prototype was to validate the requirements established for the implementation of the residents' electronic records; which enable caregivers to manage and register information about the residents' daily activities (presented in section 4.1).

Functional services.

This mobile application enables caregivers to have an inventory control, medication intake alerts, and to register as well as consult daily activities (feeding, hygiene, etc.).

A desktop application enables general staff to manage the elders' care process including the scheduled medication, meals, and recreational activities. Also the application allows sending messages to specific users for specific tasks. Physicians have the historic electronic medical records integrated in one application.

Non-functional services.

Daily activities logs of each resident are stored in a data base.

For security reasons, we proposed the use of roles (Insight \#8, Technical requirement \#8.1); depending on the role, management and retrieval of information can be granted or denied. In this application, managers, caregivers, geriatricians, physicians and nurses can have a personal account to access the system. 
Implementation details.

This prototype is intended to be unobtrusive, proactive and requiring a small cognitive load (Insight \#1).

The mobile application was installed in an android smartphone; with this the caregiver can record the activities performed by each resident using Near Field Communications (NFC) tags or a single touch. The caregivers receive notifications on the device (Insight \#3, Technical requirement \#3.1), as a reminder, if a resident should be medicated.

The desktop application was developed in Java and it was installed in a touch display computer.

\section{Advantages.}

SSAMI was used for registering the activities of the residents (Technical requirement \#7.1) instead of paper records. It was designed to interact with NFC tags (see Fig. 6a). Those tags were placed all around the residence to reduce the time of interaction with the device. This way the caregivers were creating an activity log with the activities of each resident (see Fig. 6b). For instance, when a resident went to the infirmary to have his/her blood pressure checked, the nurse would scan one of the tags in the wall to record this activity. These activity logs were probed useful as an easy, fast and in real-time input registering process for the predictive schedule.

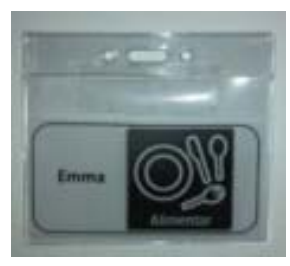

a)

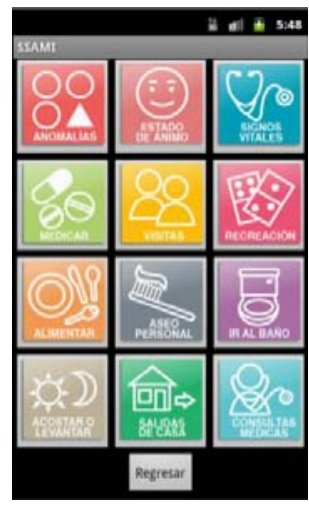

b)

Fig. 6. NFC tags placed in the residence a) and a view of the SSAMI application b).

\subsubsection{Prototype 2. SMAMI.}

Purpose.

The main objective of the development of this prototype, SMAMI, was to validate the requirements established for informing caregivers with notifications through the monitoring of residents whereabouts and risky situations in real-time, gathered from the study in-situ.

\section{Functional services.}

This prototype was designed for continuously monitoring residents' mobility, to determine their location, and for sending real-time notifications to caregivers. The data from location sensors and accelerometers were collected in a central server. From the collected data it was possible to detect risky locations (Insight \#2, Technical requirement \#2.1) and from the motion sensors data, a possible fall can be detected (Technical requirement \#2.2, Insight \#3, and Technical requirement \#3.1). 
An important component of SMAMI is a Wireless Sensor Network (WSN). Therefore, a large number of fixed sensors (fixed nodes) was installed in strategic areas of the residence to sense residents. The residents wore a sensor with wireless communication (mobile node) at the waist. The fixed nodes determined the location of mobile nodes and retransmitted data. The mobile nodes have an inertial unit composed by three-axis accelerometer; these data also travel through the network to the base node in the server. The server processes the received data and recognizes the activities processing the accelerometer data using the well-known decision tree algorithm (Jeong et al. 2007). Furthermore, the recognized activities and location are combined in the server to detect risky situations, and immediately send notifications to caregivers, for example, when residents entered restricted areas (e.g. the kitchen or the infirmary) or in case they suffered a fall (see Fig. 7). The notifications service works with mobility, places, duration and the hour of the day. Also, a decision tree (Rokach \& Maimon 2008) was used to decide when to send notifications and inform caregivers about the risky situations.

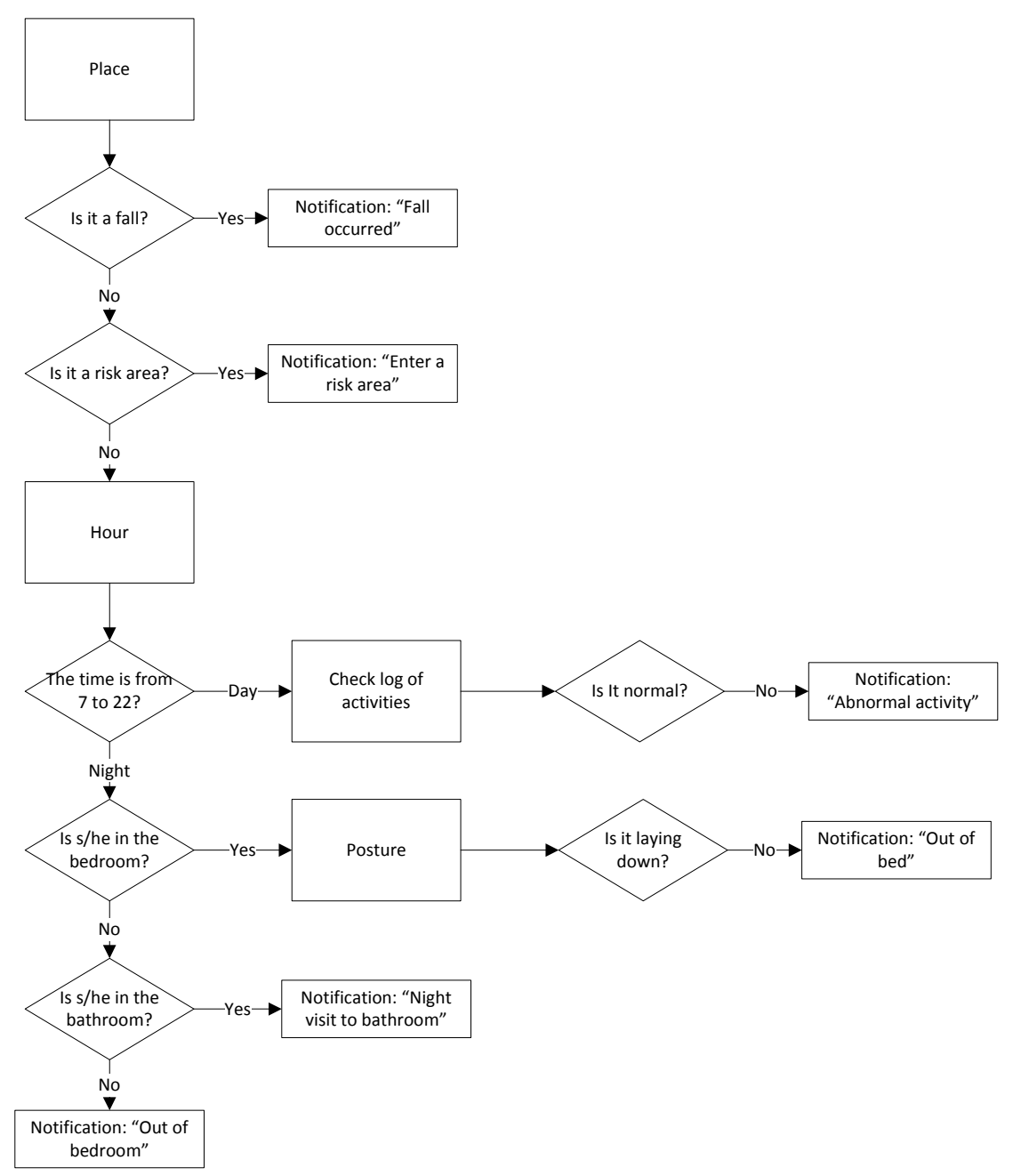

Fig. 7. Decision tree used to send notifications to caregivers. 
The prototype was developed as an android application that showed caregivers the location of the residents in real time (Fig. 8a), and also alerted them when a dangerous situation occurred (Fig. 8b). It was important to be able to validate that the notifications were done on time; for instance when a fall occurs is critical, therefore notifications should be provided just in time (Technical requirement \#2.2).

Non-functional services.

The data from location sensors and accelerometers was collected in a central server and a mechanism to improve the accuracy of the locations in real-time was developed. The accelerometer data was fed to a process recognizing postures and launching notifications.

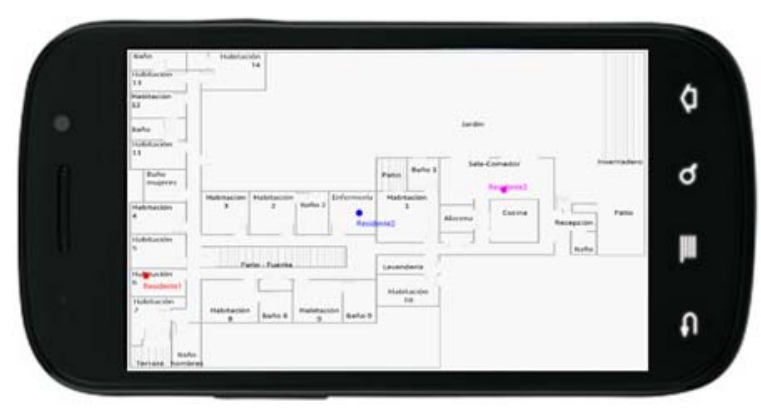

a)

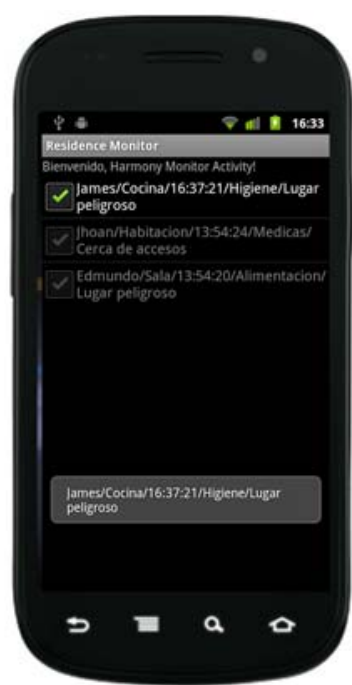

b)

Fig. 8. SMAMI system showing: a) location of residents and b) notifications.

\section{Implementation details.}

An indoor location system was deployed, based on radiofrequency triangulation with a sensor network. Also, accelerometry data was used to infer elders' activities (sitting, lay down, etc.). Both location and activities were reported to an Android device available to the caregiver.
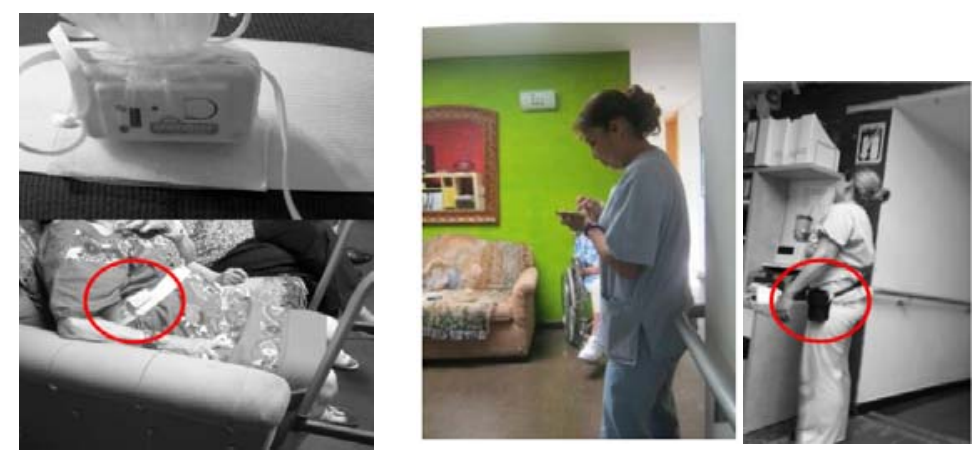

Fig. 9. Residents and caregivers using the prototypes (SSAMI and SMAMI). 
Advantages.

Before using the prototypes, elders' monitoring had to be done by following them at all times. SMAMI worked as a surrogate "follower", informing the caregivers of the whereabouts of the residents at all times, notifying them in case of a risky situation (Technical requirement \#3.1).

The architecture of both prototypes was based on the client-server and publishersubscriber paradigms, enabling complete functionality for real-time notifications and up-to-date information in the server side.

\subsubsection{Evaluation}

During four weeks both prototypes were evaluated in-situ in one of the residences, to validate the technical requirements, the feasibility of the implementation and the usefulness of the elements contained in each application (Fig. 9 illustrates residents wearing sensors and caregivers using the mobile application). Besides this, they were used daily by the caregivers for five months after the evaluation, demonstrating the need and usefulness of this kind of assistive technology.

As just mentioned, one of the aims of the in-situ evaluation in the geriatric center was to measure the perception of use of the functionality of the prototypes and, their usefulness; this was done with 11 caregivers and 12 residents. Also, data was acquired by means of 12 hours of observation, 11 interviews and 2 focus groups. These results validated the usefulness of activity's records (Technical requirement \#7.1) and provided complementary information on some aspects not covered in the initial requirements taken into account in the design of the prototypes, such as the excessive number of notifications (Technical requirement \#3.1 and Technical requirement \#9.1). Furthermore, it provided new design insights and technical requirements for the final design of the predictive schedule with a clearer view of the components that a system for elders care in nursing homes should contain; for instance, the time to record activities with the application (Technical requirement $\# 13.1$ ).

The experiences, comments and opinions from the interviews and focus group of caregivers, from the in-situ evaluation, were analyzed using open coding. The above analysis was used for establishing additional requirements for the predictive schedule. These are described next.

Insight \#9. A large number of notifications could be annoying and stressful for caregivers when they are performing other task, there should be a small number of relevant notifications.

Technical requirement \#9.1. A module to arbitrage notifications and alerts in real-time should be included.

Technical requirement \#9.2. The system should detect when the caregiver is busy.

One result from the analysis was that constant notifications are annoying; a mechanism for prioritizing and controlling the number and frequency of notifications 
was needed. "The notifications were obnoxious because they were constantly ringing" [Caregiver 7].

Insight \#10. A system that informs caregivers increases the awareness of residents and caregivers perceived improvements on the care process.

Technical requirement \#10.1. The interface should include different formats to inform caregivers about complex information for each resident with an appropriate metaphor.

Through the evaluation of the systems, we confirmed the system usefulness and ease of use; the caregivers were more aware of what the residents were doing and where they were at any given moment. In the focus group this feature was consistently perceived as enabling better care of residents. "When she (the resident) was wearing that tracking device, we just go to that map where they show they are and you look over there and it's easy to track them" [Caregiver 4]. In the focus group this feature was consistently perceived as enabling better care of residents.

Insight \#11. A failure detection module is needed.

Technical requirement \#11.1. The system should recover automatically from failures. A failure detection module should monitor battery, connection status, and the reactivity of the actual application.

Insight \#12. Data sources should be diversified and redundant to make the system reliable.

Technical requirement \#12.1. The system should include a module to interpolate lost data from historical records, redundant sources, etc.

During the evaluation there were some technical issues present, such as miscommunication between the wearable sensors and the server, and between the mobile phones and the server. Also the physical infrastructure of the residence was not conductive for a good performance of the devices used. The results from the analysis of the interviews performed during the evaluation, established that there were incomplete records due to missing data lost as a consequence of the technical problems. It is important to consider these common errors in the design of real care systems, where reliable data are important because they are the basis to extract and infer further information ("We (the caring staff) need technical support, if the system fails who is going to restart it" [Caregiver 10]).

Insight \#13. A mechanism to avoid the constantly text input by caregivers is needed.

Technical requirement \#13.1. The system should be design to diminish the caregiver constant manual registering.

Even if the systems are aimed at helping caregivers, the perceptions were disparate about the usefulness of the system. It was found that not all the caregivers perceived a substantial advantage between the use of paper and electronic logging 
due to the continuous 'writing' registering that some caregivers considered was the same thing. "In the notebook (referring to the paper logs) it was just writing, it is essentially the same (with the application), but using technology" [Caregiver 8].

By means of the in-situ evaluation we collected data about the residents and caregivers. The data recorded during the evaluation consisted of the activity logs of the residents, notes from caregivers, location and accelerometers from wearable and environmental sensors. Also, we obtained a list of socio-technical issues which result useful when conducting a naturalistic long-term evaluations (Soto-Mendoza \& Garcia-Macias 2014). With the results of the elements established during the data analysis and the requirements obtained from the evaluation of the prototypes, the design of the predictive schedule, PRESENCE, was established. Next we focus on the design of PRESENCE.

\section{PRESENCE, A PREDICTIVE SCHEDULE}

The results obtained from the analysis of the data gathered from the collected activity logs, data from sensors, caregivers' notes, interviews, observations, focus group sessions and, the in-situ evaluation of the elements validated with the prototypes, naturally lead us to envisage a more challenging objective, a predictive scheduling system.

Activity recording is a necessary task for caregivers in geriatric centers; therefore, it is important to provide appropriate support for this task that facilitates and minimizes the workload of caregivers (Technical requirement \#13.1). This was validated with SSAMI. On the other hand, notifications probed to be useful (Technical requirement \#3.1); however, the mechanism used for alerting notifications was not adequate for this purpose (suggested with SMAMI), as they became annoying. In general, from the evaluation of both prototypes we validated in-situ the general requirements for the final design of PRESENCE. We incorporated the validated, corrected and improved design characteristics of each prototype, which resulted relevant to users, in the design of the predictive schedule. In particular, from the evaluation of the activity recording we established the relevance of exploiting the data from the logs (which are a reliably and accurate source of information) to discover hidden patterns to detect risky situations and abnormal deviations. For this purpose, we propose a predictive module to be the core of PRESENCE. In the next sections, we present the complete design, architecture and functionality of the predictive schedule.

\subsection{Designing of PRESENCE}

The final application turned into a predictive schedule for caregivers (Technical requirement \#13.1) that we called PRESENCE. The predictive mechanism will provide anticipated support for caregivers' tasks related with residents' activities by analyzing the activity records and detecting patterns. Therefore, the main core of PRESENCE is based on a predictive module. PRESENCE is a front-end application; the general view of the predictive system and the description and functionality of each component is described next.

One of the main characteristics of the evaluated prototypes was the sending of notifications to caregivers (Insight \#10). However, as a result of the evaluation we 
found that a big number of notifications are overwhelming for caregivers (Insight \#9). Therefore the final design of the predictive schedule has an arbitrage algorithm to prioritize notifications to avoid increasing the stress of caregivers at work (Insight \#13), only urgent notifications would be forwarded to nurses or caregivers.

The design of PRESENCE includes a new module for the analysis of activities in greater detail (Technical requirement \#12.1). The analysis aims at providing a higher level of abstraction on the state of a resident. One extreme example would be to say, "Mr. Robert is $O K^{\prime}$. As the abstraction level becomes higher, the work of the caregiver becomes simpler; but it may not be easy to implement. We need to compromise between functionality, design requirements and the ability to implement them. We found a compromise where the caregivers do not have to record everything manually, and the system will recommend the next activity to follow; waiting for the manual input of the caregiver agreeing or not with one touch.

With SSAMI (section 4.3) the use of a roles' manager to use the application was evaluated. However, considering roles to access the system is not enough. A new requirement for the design of PRESENCE is that there should be access policies to the data at different levels, taking into account the data types, so that external entities, such as the residents' external physicians, have a secure access to the residents' information (Insight \#8, Technical requirement \#8.1).

The design of the predictive schedule should cover an error recovery and fails support mechanism, as described (Insight \#11, Technical requirement \#11.1). For this reason, we consider improving location algorithms, falls detection and activity recognition. Moreover, the caregivers' assessments must be incorporated to have more accurate measures. The predictions will be more reliable and can be assessed with the help of the caregivers' annotations (Technical requirement \#12.1).

\subsubsection{PRESENCE architecture}

The general architecture of PRESENCE is presented in Fig. 10. It is a three-tier architecture: server, client, and source, as we used in the design of the prototypes. The server contains the principal functions: the services and data manager component to cope with the data and information received from the different sources of data, and to organize the diverse services offered by the system (e.g. notifications); the predictive module is responsible for the data analysis, and will perform data fusion when they come from different sources; it also includes pattern discovery and an inference engine in this component. The component for controlling the emission of notifications is also depicted. The privacy component will manage all the privacy policies; the incorporation of dissimilar data sources could increase the complexity of the data privacy policies, for this reason a semantic knowledge component was proposed as a part of the system with the data stored in a semantic repository. Concerning the sources, different elements, sensors or applications can be incorporated; all sources should be part of the data repository. Finally, all the user interfaces and mobile applications will be found in the client's side. 


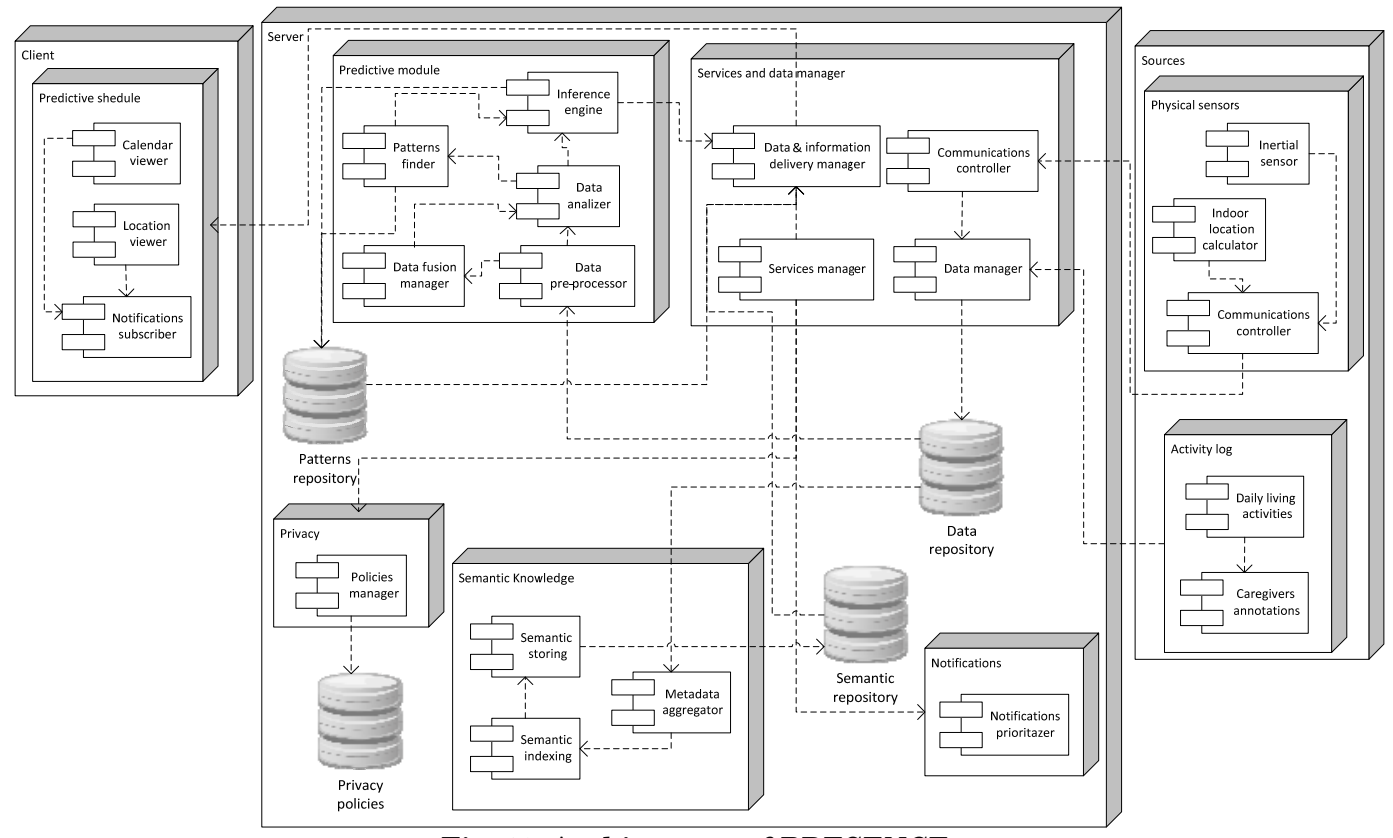

Fig. 10. Architecture of PRESENCE.

Other modules could be added in the previous schema, this poses a scalability challenge to the data manager, which should be capable of recognizing, analyzing, generalizing and establishing data privacy policies to a new data source. This can be crucial when a large amount of data is stored and ready to be processed for making inferences, reporting messages and in general to be of use. For instance, when each resident wears a small device to monitor the activity level (e.g. a Shimmer motion unit); the single data source of this device should be multiplied by the number of residents, the number of devices and, the sample rate.

The data fusion manager will use the degree of confidence of each data source to output the most reliable reading. For example, it is advisable to give a strong confidence to a caregiver annotation if the data represents the mood of a resident; but a sensor is more reliable to verify the heart rate or the temperature of a resident. The data fusion manager will construct a data warehouse by selecting and aggregating data coming from different sources (sensors or caregivers) and stored in the data repository. To do this, ETL techniques (Extract, Transform, Load) can be used. There exist open source tools like the one proposed by Talend ${ }^{1}$ that may facilitate this task.

The services manager was proposed to control the flow of each input or output service (e.g. sensors, notifications). When a notification emerged the services manager uses the notifications prioritizer to know the urgency of the message. This module is also responsible for data reliability, with subroutines handling communication protocols, such as CoAP (Constrained Application Protocol) and MQTT (Message Queuing Telemetry Transport) which have more tolerance to

\footnotetext{
${ }^{1}$ Talend Open Studio for Data Integration (http://www.talend.com/download?qt-download_landing=3\#qtdownload_landing)
} 
communication faults and latency. Uninterruptable power sources are provided to support static sensing infrastructure.

Caregivers will continue registering residents' activities. The data repository will store both: activities logs and sensor data. The inference engine will take the data from the repository and process it to provide structure. The data fusion will mix the data sources to analyze and discover patterns in the activities. The patterns found will be stored in the patterns repository.

Additionally, the data management and control module should be supervised by the privacy policies to enforce physical devices and personnel to respect the privacy agreement of the data. Preserving privacy of residents is very relevant because data collected by PRESENCE is very sensitive. It is important to establish a personal privacy policy for each resident (helped by the corresponding tutor) that should be preserved by the schedule. Such policies will describe which data is collected, whom can access them, for what purposes they are collected, for how long they will be stored (data should not be stored forever), etc. Policies will follow well-known guidelines and legislations as the ones proposed by the $\mathrm{OECD}^{2}$ (Organisation for Economic Co-operation and Development), by the $\mathrm{CNIL}^{3}$ (National Commission on Informatics and Liberty), or by HIPAA $^{4}$ (Health Insurance Portability and Accountability Act).

The data collected during a long period of time could be analyzed in different manners; therefore the results of the analysis will be diverse and will have a lot of applications. The data acquired and processed could represent complex concepts, hence we propose a complete representation through semantic technologies. The relationships and causalities could be modeled using ontologies. The relationship between data and entities is modeled with RDF (Resource Description Framework); the same could be used for the interactions with smart objects. These concepts come from the Internet of Things (IoT) and semantic web, in the proposed system they are contained in the semantic knowledge module to be combined with the machine learning techniques used inside the inference engine.

In the client side we propose a component to manage all the user interfaces of the system, namely desktop and mobile applications. Our mobile application, the predictive schedule, would be part of this node and it will show caregivers the digested data resulting from the inference analysis, the activities and location as the initial prototypes did.

\subsubsection{Appearance and functionality}

The predictive schedule is designed to look mostly as a common calendar (see Fig. 11a). The calendar is fed with two types of information; on the one hand the actions from the medical protocol (medication, therapy, activities, etc.) are inserted as mandatory (see Fig. 11b); on the other hand it is fed with the information generated from the inference module, the complementary actions following a mandatory task

${ }^{2}$ (http://www.oecd.org/internet/ieconomy/oecdguidelinesontheprotectionofprivacyandtransborderflowsofpers onaldata.htm)

${ }^{3}$ http://www.cnil.fr/english/

${ }^{4}$ https://www.privacyrights.org/content/health-privacy-hipaa-basics 
are suggested. In this way, caregivers would know what tasks are pending and the tentative deadlines for them. The task will be considered as "executed" when the caregiver clicks on it. Caregivers can visualize the activities or tasks scheduled in a month, week or day basis. Also the application enables caregivers to consult the activities of all residents per day, or filtering the activities by resident. The predictive schedule is dynamic, meaning that if a series of events deviate from the usual, predictions will be recomputed and the schedule will be updated accordingly.

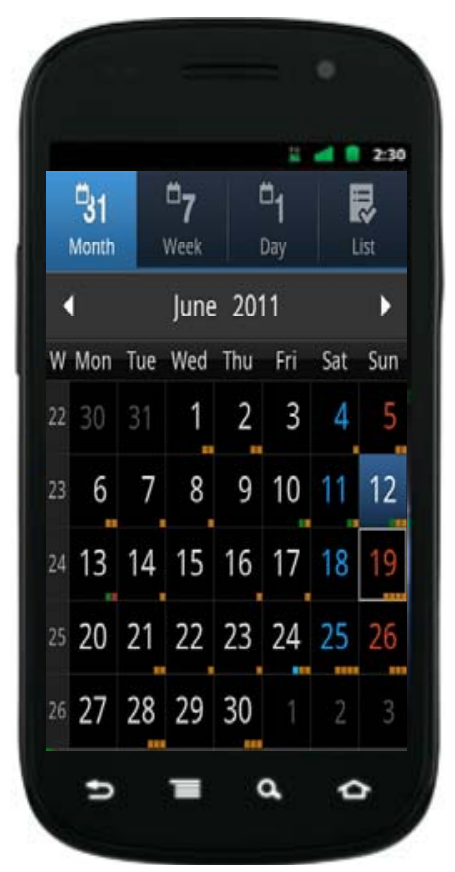

a)

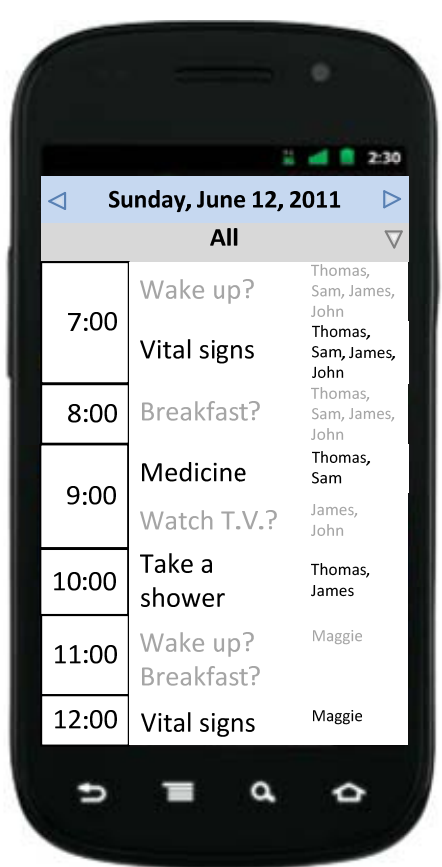

b)

Fig. 11. (a) Appearance of the predictive schedule and (b) the information it presents.

Each prediction has its proper level of importance. Actions from the medical protocol are mandatory and have the highest level of importance; for example not giving some medicine to a resident has not the same level of importance than a resident who does not receive visits one day. The mandatory part of the schedule and the optional complements are blended in the interface. We assigned priorities to each prediction related with an activity. As time goes by, the schedule evaluates and contrasts the expected activities with those that have occurred. Finding a perfect match indicates that everything is according to the schedule. When a mismatch is detected the application notifies caregivers about the deviation of the plan (see Fig. 12a). Depending on the importance of the deviation the caregiver will receive a silent, normal or urgent notification. This is to avoid annoying the caregiver with unimportant updates. However, if the caregiver wants to check for a particular resident, all the notifications will be there for him/her to peruse. Urgent notifications, for example when a vital medicine was not given to a resident (see Fig. 12b), will not go away until they are attended. 


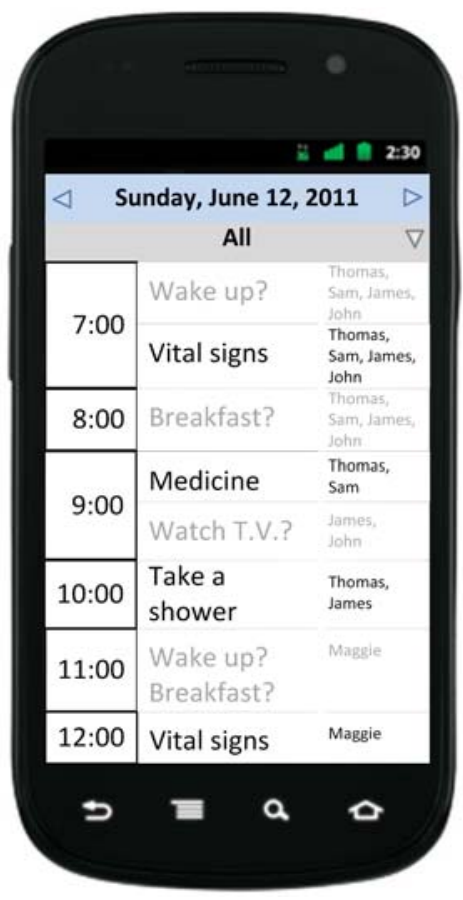

a)

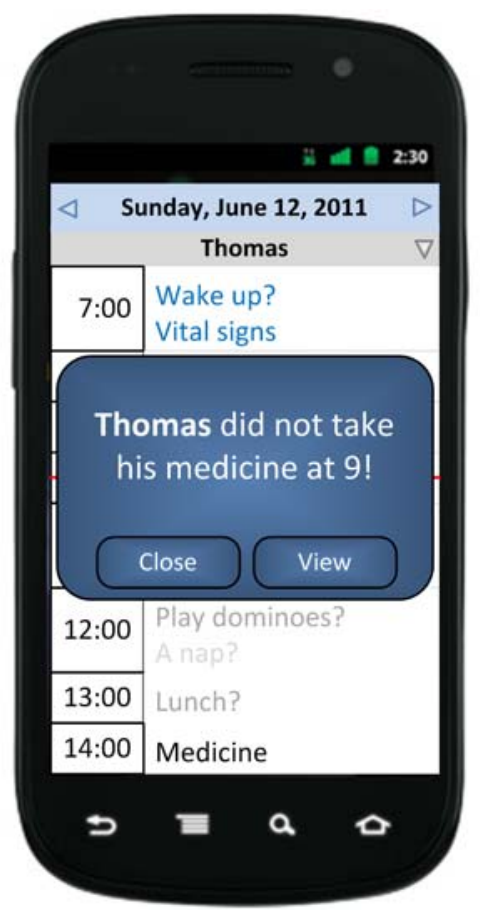

b)

Fig. 12. (a) List of suggestions and (b) notifications presented by the predictive schedule.

The data collected from the in-situ evaluation of the prototypes was manually mined and processed to find usual and unusual patterns in the states of each resident. For instance, if one day the caregiver observes that the resident is having breakfast before someone read his vital signs, it would be an unusual pattern because it is mandatory for a caregiver to take the resident's vitals immediately after s/he gets out of bed. Another example is when a resident has the same succession of states, day after day, during a couple of months; then suddenly the resident changes something in his routine, the schedule should be capable of detecting this deviation.

Next, we present a feasibility analysis of the predictive module of PRESENCE based on the activity data logs analysis and its main components.

\subsection{Feasibility analysis of the predictive module}

We explored the viability of the implementation of the predictive module through an activity logs analysis. We refined, organized, analyzed and processed data from activities' logs gathered during the evaluation to find correlations and patterns among them, which could be used to feed the predictive schedule. These logs are mainly related with activities of the residents, from which we defined their different states. Each state indicates the general condition of the resident at a given moment. Using these states we obtained regular transitions among their states and built a sequence of states in a normal day. The functionality of each component of the predictive module is presented next. 
Data pre-processor

Its task is to clean and format the activity logs of residents registered by caregivers and the data collected by physical sensors. We created an activity model to formalize the concepts and entities found among data. For this reason, we redefined the activities and characterized states of the residents to build the inference engine based on a finite state machine. The defined states were:

- Sleeping

- Definition: the resident is in his bedroom or in the living room and presents a specific movement pattern. Usually, this state occurs at night but it may also occur in the morning or afternoon.

- Measured by: the caregiver based on a physical inspection, motion sensors, pressure sensors and/or proximity sensors.

- $\quad$ Eating

- Definition: the resident is eating by himself or with the caregiver's help. The residence can usually be in his bedroom or dining room. The residents must eat at least three meals per day.

- Measured by: the caregiver or cooks, or by location, pressure or proximity sensors.

- $\quad$ Socializing

- Definition: when a resident interacts or talks with another person.

- Measured by: caregiver's visual observation, proximity sensors or voice activity detection.

- Awake

- Definition: the resident just got out of bed.

- Measured by: the caregiver based on resident's physical inspection, or motion sensors, pressure sensors (located under the bed) or proximity sensors.

- Outside

- Definition: when the resident attends outdoor activities, outside the residence, he goes to a play in a theater, the movies, beauty parlor, etc.

- Measured by: caregiver's visual observation, or proximity or location sensors.

- Cleaned

- Definition: when the resident is taking a shower or is in the bathroom for changing the diaper, washing hands and brushing teeth.

- Measured by: the caregiver can inspect the resident, location sensors.

- Medicated

- Definition: when the caregivers or a nurse gives a medicine to the resident.

- Measured by: caregivers' records.

- Entertained

- Definition: the resident participates in a group dynamic, a playful activity in the dining room, living room or in the garden, watches T.V. or plays dominoes. Usually there is a group of people together in the same place at the same time. These activities occur between meals. 
- Measured by: caregiver's direct visual inspection or by proximity or location sensors.

Once the activities data is transformed into states, the data is prepared to feed the data fusion manager and the data analyzer.

\section{Data fusion manager}

This component discriminates or combines data based on their reliability level in order to improve the accuracy of the inferences or to complete missing data. We can observe, from the previous definitions, that either caregivers or different types of sensors can gather information that can be used to infer the states of a resident. However, there exist great challenges in sensing accurate measures. For example, detecting a gathering of people interacting with each other can be very tricky for sensors; when they are located in common areas the information gathered comes from several sources.

\section{Data analyzer}

This component takes the state representation from the data pre-processor and transforms it into a state's transition matrix (see Fig. 13).

\begin{tabular}{|c|c|c|c|c|c|c|c|c|}
\hline 6 a.m. to 2 p.m. & $\begin{array}{l}\stackrel{00}{0} \\
\frac{0}{0} \\
\frac{0}{\Delta}\end{array}$ & 苞 & $\begin{array}{l}\vec{D} \\
\mathbb{E} \\
\text { U] }\end{array}$ & $\begin{array}{l}\frac{\mathscr{v}}{\pi} \\
\frac{\pi}{3} \\
\end{array}$ & 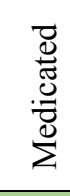 & 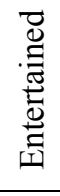 & $\begin{array}{l}\frac{0}{0} \\
\frac{0}{0} \\
0 \\
0\end{array}$ & 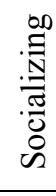 \\
\hline Sleeping & 0 & 0 & 0 & 2 & 1 & 0 & 0 & 0 \\
\hline Eating & 2 & 5 & 19 & 4 & 54 & 1 & 0 & 0 \\
\hline Cleaned & 0 & 12 & 16 & 0 & 7 & 0 & 1 & 0 \\
\hline Awake & 0 & 47 & 12 & 2 & 2 & 0 & 0 & 0 \\
\hline Medicated & 1 & 19 & 32 & 18 & 235 & 0 & 0 & 0 \\
\hline Entertained & 0 & 0 & 0 & 0 & 0 & 0 & 0 & 0 \\
\hline Outside & 0 & 1 & 0 & 0 & 0 & 0 & 0 & 0 \\
\hline Socializing & 0 & 0 & 0 & 0 & 0 & 0 & 0 & 0 \\
\hline
\end{tabular}

Fig. 13. State transition matrix of a subject (resident) telling the story of her usual mornings.

This arrangement, although simplistic, is quite informative. We demonstrate its functionality by processing the logs of 14 subjects (residents marked as S1 to S14) to obtain the transitions between states. Then we counted the number of times one state follows another, in Fig. 13 we highlighted the transitions with the highest frequency. In this process data were split into 8 hours chunks depending on the timestamp. The first chunk includes activities between 6 a.m. and 2 p.m., the second between 2 p.m. and 10 p.m. and the third from 10 p.m. to 6 a.m. of the next day. In general, changes of state occurred often from 6 a.m. to 2 p.m. because residents and caregivers performed more activities (e.g. taking showers, changing clothes, taking breakfast, and having medication) at the beginning of the day. Then a decrease in transitions was observed during the following two time slices (afternoon and night) 
(Fig. 14). For example, resident 14 (S14) has the largest number of transitions at any given time slice. He is also the one with the most needs of care, because he has advanced Parkinson's disease. Subject S14 cannot perform almost any activity without assistance since he cannot move, walk or even talk. In the case of subject S12, the transitions in the afternoon were more than in the morning and night; in this case the resident had dementia and he usually woke up later than the rest, and his activities started late, which implies a shift in the activity period, coinciding with everybody else in the afternoon. When residents went to sleep the number of transitions was significantly reduced.

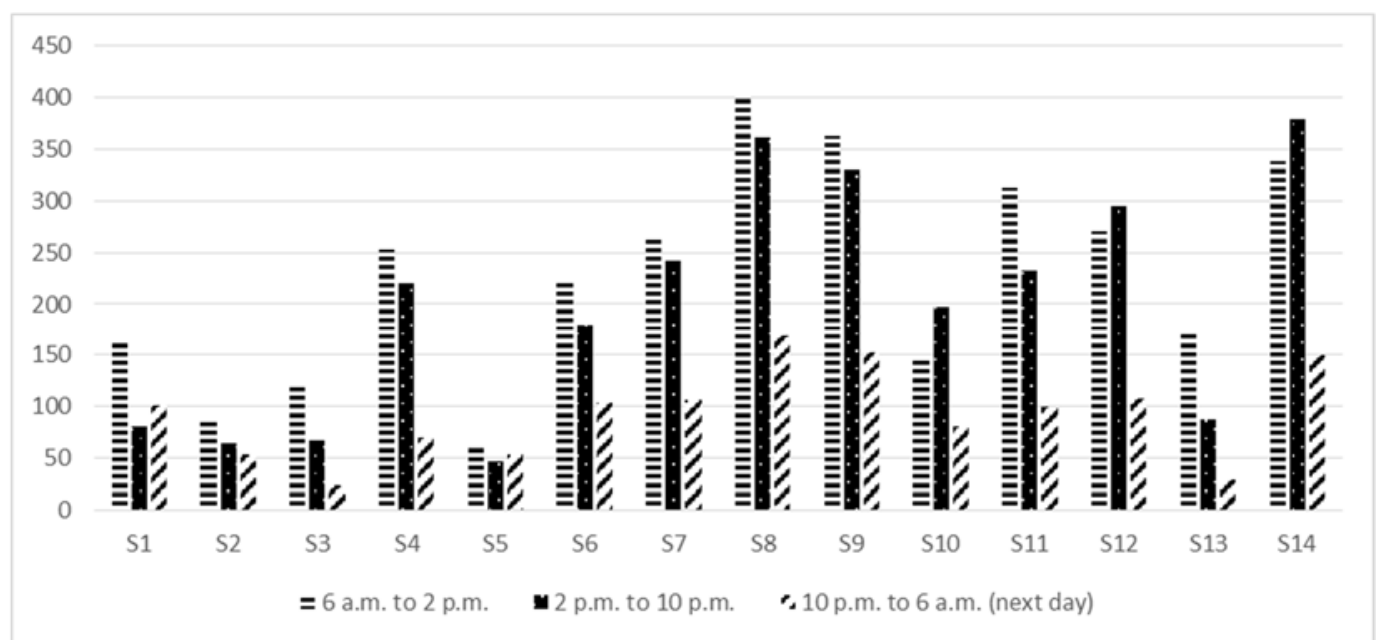

Fig. 14. Number of states transitions (frequency) of each subject (resident) at morning, afternoon and night (without medication).

The transitions matrix might be used to predict the level of care needed by each resident, which is highly valuable information for planning purposes, because the residents had different medical conditions and hence the needs of each one are variable. Another application for the transition matrix is to separate residents into groups to assign a more skilled caregiver for the more demanding group, i.e., the number of transitions is a very consistent indicator of the independence level. In other words, residents who can still do things by themselves have a smaller number of transitions than residents who need more assistance.

\section{Patterns finder}

The component analyzes the state's log of each resident looking for patterns or sequences of states within a specific period of time. We show its functionality analyzing the states performed for each resident on weekdays versus weekends. From this analysis, we could observe a difference in the transitions between states. During weekdays the level of activities is higher than during weekends: This happens because in some cases the family visits and takes their relative out or when they spend a weekend outside. The finder uses naïve transition matrix (see ¡Error! No se encuentra el origen de la referencia.) to detect usual and unusual days or periods of time for a given resident. The matrix also serves as the basis to construct a useful narrative to describe the day of a resident and to inform the relatives, an example could be the following: 
During the last 4 months, mornings have been usual for Juanita. She begins the day asleep and wakes up around 7 a.m. She receives her medicine and after she changes her clothes, she goes to the dining room to have breakfast. Later, the caregiver gives her medicine and, afterwards, assists in Juanita's shower. When Juanita is clean she takes a walk and then she eats again because she has very good appetite all day.

The boldface sections on the above narrative, comes from the most likely transition in the matrix, after some polishing for readability. For each resident and each transition, a phrase is added. Please notice that the transition matrix was elaborated by hand using the available data of five months usage of the two prototypes described above. More sophisticated analysis can be done after data cleaning and training. The proposed inference module generalizes the probabilistic finite automata represented by the transition matrix.

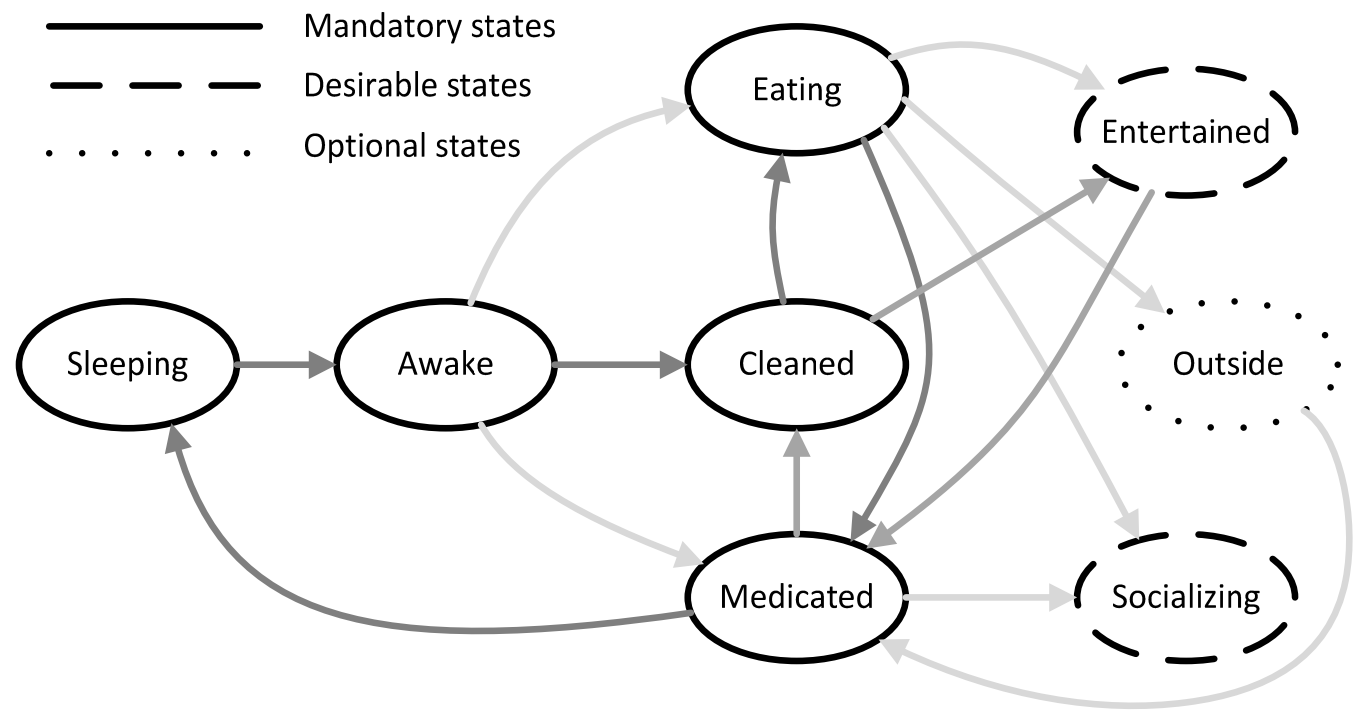

Fig. 15. Example of a sequence of states following the most probable path.

\section{Inference engine}

The inference engine uses the transition matrix and the patterns discovered to infer the next state. It creates a generalization of finite state machines to make predictions. The most well-known examples are Hidden Markov Models (HMMs) and Partially Observable Markov Decision Processes (POMDPs). The former assumes that the next state in a transition matrix only depends on the current state, while the later assumes that there is not knowledge about the current state of the system and instead maintains a distribution of the possible current states of the system. The automata (Fig. 15) we constructed from the data is just a simple example for illustration. The most important part is to define the states; once this is done, the learning process is standard. 
In our approach, predicting the next state of the resident with the highest probability is just one aspect of the problem. The schedule for a given resident may or may not consider the predicted state and, if predicted, will have a degree of compulsoriness. The external information to the model needs to be considered for the design. It is necessary to establish the compulsoriness of a state (corresponding to an activity). Another parameter would be to assign a dynamic threshold for each resident. Passing the threshold will trigger a notification for example.

The predictive schedule has to conciliate three aspects in the model: the transition probability, if the event is scheduled to happen, and the degree of compulsoriness. We have identified two cases where action is needed (e.g. sending a reminder/warning to the caregiver). Illustratively, we assume state $E 1$ is bound to happen for a certain resident with high probability and state $E 2$ is scheduled. We have two cases either $E 1$ and $E 2$ are different, or they are the same. If they are the same, then nothing is done. If they are different and the compulsoriness threshold for either the scheduled event or the event bound to happen is surpassed, then an alert is sent to the caregiver, because in either case the scheduled event is important, and is not bound to happen; or the predicted event is important and will collide with another event in the schedule.

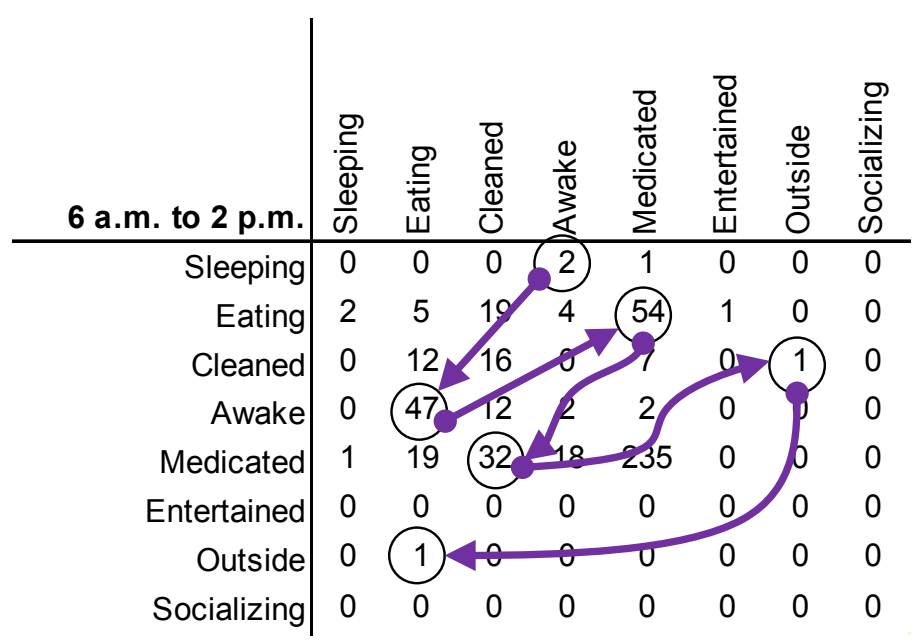

Fig. 16. Individual path following the transitions of the states.

There will be, of course, events neither scheduled nor predicted. For example a resident entering the infirmary or the kitchen. For these events there are techniques already published (Bamis et al. 2010) to prevent risky situations for the residents, they will not be considered in our design as a challenge.

The transition matrix is a way to obtain valuable information. It has the advantage of being in a very readable form and it happens to be a nice metaphor for visualizing the activity of a resident as illustrated in Fig. 16. The sequences of states indicate a daily leading path of the states of the residents (indicated by the arrows). One day of activity is a sequence of states describing the day of a resident. It is far from just guessing what the most likely next state (with high probability) is. 
The predictive module is the central part of the predictive schedule design. Sensors and caring personnel are very rich sources of data. Non-invasive activity recognition algorithms from heterogeneous data sources will be a good source of context. Modeling each resident individually is also a challenge and might provide better predictions.

Additional to this analysis, we carried out an evaluation of usage and ease of use of the final design of PRESENCE to validate these design aspects with the intended users. The results are presented in the next section.

\subsection{Evaluation session of the design}

We conducted an evaluation of the design of the predictive schedule system, PRESENCE, and its functionality with caregivers. Using scenarios and low level prototypes, we organized an evaluation session where we presented the proposed system design to a group of 4 caregivers and 2 nurses (Table 1 ).

Table 1. Respondents profile $(\mathrm{N}=6)$.

\begin{tabular}{c|c|c|c} 
& Age & Occupation & Title \\
\hline Nurse 1 & 32 & Nurse & Nurse coordinator \\
\hline Caregiver 1 & 31 & Nursing assistant & Caregiver \\
\hline Caregiver 2 & - & - & Caregiver \\
\hline Caregiver 3 & 42 & - & Caregiver \\
\hline Caregiver 4 & 47 & Teacher & Caregiver \\
\hline Nurse 2 & 22 & Nurse & Nurse
\end{tabular}

PRESENCE was presented to the participants and at the end of the session a 7point-scale Technology Acceptance Model (TAM) (Fred 1989) questionnaire was applied (see results in Table 2). In general, the caregivers considered PRESENCE as a useful application meanwhile it demands little attention and time to register. For residents the use of this type of application was perceived less useful (compared with caregivers) due to the reluctance elders may express to technology and the advance stages of theirs medical conditions.

The questionnaire included 22 assertions related with perceived usefulness and perceived ease of use. For instance, "My job will be easier with the predictive schedule" (PU), and "I'm interested in using the predictive schedule" (PEOU). Then, the comments of the sessions and questionnaires were analyzed and we obtained evidence of how the system would be perceived by caregivers when using it in their work.

The caregivers judged PRESENCE and they agreed that the anticipated information management system would be useful. They mentioned that including crying spells is very important to detect periods of depression. Also, some of them were worried about the way depression would be identified, "it is a little hard to know when somebody is depressed, unless s/he has a button or something, but it is difficult because in a moment residents are well, and the next moment they are not, it is because of their disease" [Caregiver 2]. We hypothesized that analyzing the activities' logs and the caregivers' annotations could be possible to identify these sudden 
variations. Then the system could provide a good approximation prediction of when the periods of depression will occur.

Table 2. Results of the TAM data analysis.

\begin{tabular}{|c|c|c|c|c|c|c|}
\hline & & $\begin{array}{l}\text { Question } \\
\text { Number }\end{array}$ & Mean & Std. Dev. & $\begin{array}{c}\text { Average } \\
\text { mean }\end{array}$ & $\begin{array}{l}\text { Average } \\
\text { std. dev. }\end{array}$ \\
\hline \multirow{11}{*}{ Caregivers } & \multirow{5}{*}{ Perceived usefulness (PU) } & Q1 & 5.83 & 1.47 & 5.60 & 1.36 \\
\hline & & $\mathrm{Q} 2$ & 6.33 & 0.82 & & \\
\hline & & Q3 & 6.33 & 0.82 & & \\
\hline & & Q4 & 4.67 & 1.97 & & \\
\hline & & Q5 & 4.83 & 1.72 & & \\
\hline & \multirow{6}{*}{ Perceived ease of use (PEOU) } & Q6 & 6.17 & 0.75 & 5.44 & 1.41 \\
\hline & & Q7 & 5.67 & 1.03 & & \\
\hline & & Q8 & 4.83 & 1.72 & & \\
\hline & & Q9 & 5.50 & 1.64 & & \\
\hline & & Q10 & 5.00 & 1.67 & & \\
\hline & & Q11 & 5.50 & 1.64 & & \\
\hline \multirow{11}{*}{ Older Adults } & \multirow{6}{*}{ Perceived usefulness (PU) } & Q12 & 3.83 & 1.72 & 4.39 & 2.19 \\
\hline & & Q13 & 4.33 & 2.16 & & \\
\hline & & Q14 & 4.50 & 2.26 & & \\
\hline & & Q15 & 4.50 & 2.51 & & \\
\hline & & Q16 & 4.33 & 2.34 & & \\
\hline & & Q17 & 4.83 & 2.14 & & \\
\hline & \multirow{5}{*}{ Perceived ease of use (PEOU) } & Q18 & 4.67 & 2.07 & 4.40 & 1.88 \\
\hline & & Q19 & 4.50 & 2.07 & & \\
\hline & & Q20 & 4.83 & 1.47 & & \\
\hline & & Q21 & 4.00 & 1.67 & & \\
\hline & & Q22 & 4.00 & 2.10 & & \\
\hline
\end{tabular}

* Scale items were based on seven-point Likert-type scales (1 = "Strongly disagree", 7 = "Strongly agree")

All the caregivers and nurses emphasized that the time is at essence. They do not have enough time to register the activities; therefore they will not use a system that distracts them. When the appearance of PRESENCE was presented, they commented about the facility to register activities because all the activities would be there and only caregivers would check them. Caregivers argued that it is very complicated to follow a strict sequence of activities due to the number of residents and the different tasks with each of them, but they said that PRESENCE would help them to minimize the time for activity registration.

The calendar form appearance was attractive for caregivers because they want to be able to consult previous accidents or major incidents about the residents. With the system currently in use they do not perceive the utility of registering step by step all the activities because they cannot query previously stored information. 
PRESENCE was perceived as a tool for a "rapid diagnostic of each resident" because of the design of the individual view of each resident and the flexibility to change the schedule with the activities of each elder. It was perceived as useful because they were presented in the same screen and the navigation was simple. We agree that the right path is to combine different sources of contextual information (e.g. location, mobility, social interaction, medical data, vital signs and activities performed) to create individualized profiles enabling caregivers to know the care needs of each resident. The knowledge generated will be digested and used by PRESENCE, which will "learn" from the caregivers' annotations, and will manage and organize information so that caregivers will have advanced and specific information on each resident, their habits and traditions.

The caregivers highlighted the notifications of medications, special procedures or vital signs, as the most important features related to older adult care. Most of the caregivers and nurses preferred an alarm or voice mode of notification as a reminder when they forget to do some important activities, "when by omission I forgot doing some procedure at a specific time, the system will remind me timely" [Caregiver 1].

In the system currently in use, the size of the device used for the system was detected as an issue. Sometimes the size of the smartphone makes it impossible to register the activities due to the sleep deprivation of the caregiver, the time of the night and the workload during the day. As a consequence, the time consumed for registration increases and the caregivers spend extra time after the shift has concluded "the reality is that there is not enough time" [Caregiver 3]. The use of tablets with a bigger screen and keyboard, or computers was proposed; therefore PRESENCE should offer multiplatform support.

Nurses were more focused on the incorporation of more medical information into the system, in this way diagnostics, decisions and actions could be more patientcentered, "it [the system] could incorporate some extra medical activities" [Nurse 1]. The nurse assessments are based on patterns they observed in the residents. Therefore, PRESENCE would consider incorporating these nursing patterns, "we base our diagnostics on patterns. When a diagnostic is obtained we propose a prioritized caring program" [Nurse 1]. Other medical information could be included to make the system more useful for nurses, medical staff and managers in the residence, "I believe it would be good help for nursing functions. Instead of only registering the activities, it would also implement the nursing process to support the company legally" [Nurse 2].

PRESENCE was also perceived as a training tool for new caregivers, "We [caregivers] try to teach them [new caregivers] but sometimes we are very selfish and do not teach as well as we should do" [Caregiver 4]. New caregivers just need to check and study the information in the application to know what to do with each resident and then as time goes by the new caregiver will learn, "all data must be registered in the system, and the new caregiver should study and review what she must do in her job. In practice it will be different but she would have a prior notion" [Caregiver 4]. Our system, PRESENCE, would not replace the personal and direct training of a caregiver but it would be a good first approach to the general knowledge of each resident and the whole elders' care process. 


\section{DISCUSSION}

We studied methods and processes followed during the care of older adults in nursing homes. We conducted a longitudinal field study to learn the main variables describing the quality of care and the wellbeing of residents, and we identified opportunities to lower the workload of caregivers and to improve in general the caring process.

The field study gave us a number of insights, and based on those findings we designed and implemented two prototypes of technological aids to support residents' care, namely SSAMI and SMAMI. Those prototypes aimed at increasing the awareness of the caregivers on the whereabouts of residents, and helped caregivers to record activities, vital signs, routine tasks and eventualities. The prototypes were evaluated in-situ, giving more insights and knowledge about the care process with those tools.

With SSAMI, we focus on reducing caregivers' burden through supporting the completion of their daily activities (Czarnuch \& Mihailidis 2011). Our findings complement other statements in the literature, namely that activity monitoring is an important variable to reduce subjective burden (Lexis et al. 2013). Caregivers need information of the activities and whereabouts of the elderly; this requirement is often opposed to the reluctance of elderly people to be monitored, particularly when obtrusive wearable technology is used (Rashidi \& Mihailidis 2013).

One identified source of subjective burden, even with assistive technology, was the need to record routine tasks. The predictive schedule PRESENCE, emerged from the in-situ evaluation, was a compromise between the information needs of caregivers and medical staff, the monitoring of the elderly and their reluctance to wear obtrusive technology, and the repetitive nature of the majority of caring tasks. All the modules and requirements in the design of PRESENCE (such as inference tools, wearable sensors, wireless infrastructure, etc.) are readily available in the literature or can be acquired in the case of hardware. Our main focus was the design, the perceived usefulness, and the perceived ease-of-use from the viewpoint of caregivers and medical staff. We conducted a participative design session with the users, caregivers and medical staff, to evaluate the design of PRESENCE and obtained valuable feedback to improve it (Mortenson et al. 2013).

The goal of any assistive technology for elders is to improve the elders' health. However, the challenge is to develop a technology that works for every single elder with different needs and conditions. PRESENCE is offered as a personalized solution to support the elderly and adapt the system to their personal needs (Czaja et al. 2013). PRESENCE was designed following a user-center methodology and all the design insights emerged from a field study which enables to collect real contextual information related with elders' care process in geriatric centers.

In the evaluation, the nurses and caregivers considered the inclusion of more medical information, such as the nursing process; these observations suggest the need of continuous training for caregivers to provide more professional and specialized care, and encourage the confidence in the task they performed (Bourbonniere \& Evans 2002). The final design helps caregivers to register events faster, to be aware of the residents' needs and in general to increase the quality of the 
care process with a small cognitive load. The integration of caregivers' annotations and sensor data is informative; it satisfies the required predictive features and is simple enough to be integrated seamlessly in the caring workflow.

\section{CONCLUSIONS}

Research in assistive technology has focused on shifting the need of human caregivers and substituting them with technological aids to leverage independent living of older adults. This is certainly a plausible path in the future, in a healthier society with a small prevalence of chronic, incapacitating diseases. Geriatric centers, on the other hand, will be here for a couple of generations. They have an urgent need to lower the burnout of caregivers and to improve care quality for current and future residents.

Our proposal is sensitive to current problems of geriatric centers, and takes into account the needs of the caring and medical staff; instead of planning for their dismissal with upcoming technology, we proposed mechanisms to increase the care quality, lower the burden, and contribute to the wellbeing of the residents.

The proposed system is perceived as useful by the caring personnel, medical staff, and management. The potential users are eager to try a system with the functions described to incorporate them in their daily workload. The use of PRESENCE could free caregivers from routine tasks, so they can concentrate on other activities such as leisure and socialization time with residents, and in general to give a more personalized attention.

Future work includes implementing the system and conducting a long term field study to evaluate the impact of this technology in the care facilities.

\section{ACKNOWLEDGMENTS}

Authors thank those who participated in the field study: caregivers, nurses, residents, managers and residence's staff. Partial funding for this work was provided by the Mexican National Council for Science and Technology (CONACyT), and through a scholarship provided to the first and last author.

\section{REFERENCES}

Aloulou, H. et al., 2013. Deployment of assistive living technology in a nursing home environment: methods and lessons learned. BMC medical informatics and decision making, 13, p.42.

Archer, N. et al., 2011. Personal health records: a scoping review. Journal of the American Medical Informatics Association : JAMIA, 18(4), pp.515-22.

Bamis, A. et al., 2010. The BehaviorScope framework for enabling ambient assisted living. Personal and Ubiquitous Computing, 14(6), pp.473-487.

Bardram, J.E., 2008. Pervasive Healthcare as a Scientific Discipline. Methods of Information in Medicine, 47(3), pp.178-185.

BeClose, 2013. BeClose. Available at: http://beclose.com [Accessed July 2, 2013].

Blumenthal, D. \& Tavenner, M., 2010. The "Meaningful Use" Regulation for Electronic Health Records. New England Journal of Medicine, 363(6), pp.501-504.

Borenstein, J. \& Pearson, Y., 2010. Robotic caregivers: Harbingers of expanded freedom for all? Ethics and Information Technology, 12(3), pp.277-288.

Botia, J. a., Villa, A. \& Palma, J., 2012. Ambient Assisted Living system for in-home monitoring of healthy independent elders. Expert Systems with Applications, 39, pp.8136-8148.

Bourbonniere, M. \& Evans, L.K., 2002. Advanced Practice Nursing in the Care of Frail Older Adults. Journal of the American Geriatrics Society, 50(12), pp.2062-2076.

Bravata, D.M. et al., 2007. Using Pedometers to Increase Physical Activity A Systematic Review. American Medical Association, 298(19), pp.2296-2304. 
Checkland, P.B., 1989. Soft Systems Methodology. Human Systems management, 8(4), pp.273-289.

Coeckelbergh, M., 2010. Personal robots, appearance and human good: A methodological reflection on robots. International Journal of Social Robots, 1(3), pp.217-221.

Cornejo, R., Hernández, D. \& Favela, J., 2012. Persuading older adults to socialize and exercise through ambient games. In 2012 6th International Conference on Pervasive Computing Technologies for Healthcare (PervasiveHealth) and Workshops.

Curtis, B. et al., 1992. Process modeling. Communications of the ACM, 35(9), pp.79-90.

Czaja, S. et al., 2013. Older Adults and the Adoption of Healthcare Technology: Opportunities and Challenges. In A. Sixsmith \& G. Gutman, eds. Technologies for Active Aging. Boston, MA: Springer US, pp. 27-46.

Czarnuch, S. \& Mihailidis, A., 2011. The design of intelligent in-home assistive technologies: Assessing the needs of older adults with dementia and their caregivers. Gerontechnology, 10(3), pp.169-182.

Davenport, T.H., 1993. Process Innovation. Harvard Business School Press.

Decker, M., 2008. Caring robots and ethical reflection: The perspective of interdisciplinary technology assessment. AI \& SOCIETY, 22(3), pp.315-330.

Fatima, I. et al., 2013. A unified framework for activity recognition-based behavior analysis and action prediction in smart homes. Sensors, 13, pp.2682-99.

Fayyad, U., Piatetsky-shapiro, G. \& Smyth, P., 1996. From Data Mining to Knowledge Discovery in Databases. AI Magazine, 17(3), pp.37-54.

Fennell, M.L. et al., 2010. Elderly hispanics more likely to reside in poor-quality nursing homes. Health affairs (Project Hope), 29(1), pp.65-73.

Fred, D., 1989. Perceived Usefulness, Perceived Ease Of Use, And User Acceptance of Information Technology. MIS Quarterly, 13(3), p.319.

Glaser, B.G. \& Strauss, A.L., 2012. The discovery of grounded therory: strategies for qualitative research 7th ed., New York, New York, USA: Aldine Transaction.

Havasi, F. \& Kiss, Á., 2008. Ambient Assisted Living in Rural Areas: Vision and Pilot Application. In AmI 2007 Workshops, CCIS. pp. 246-252.

Hossain, M.A., 2014. Perspectives of human factors in designing elderly monitoring system. Computers in Human Behavior, 33, pp.63-68.

Hossain, M.A. \& Ahmed, D.T., 2012. A Human Caregiver Support System in Elderly Monitoring Facility. In 2012 IEEE International Conference on Multimedia and Expo Workshops. Ieee, pp. 435-440.

Jeong, D.-U., Kim, S.-J. \& Chung, W.-Y., 2007. Classification of Posture and Movement Using a 3-axis Accelerometer. In 2007 International Conference on Convergence Information Technology (ICCIT 2007). Ieee, pp. 837-844.

Kaluža, B. et al., 2012. Context-Aware MAS to Support Elderly People. In Proceedings of the 11th International Conference on Autonomous Agents and Multiagent Systems (AA-MAS 2012). pp. 14851486.

Kaluža, B. et al., 2011. Intelligent monitoring of the elderly in home environment. AAAI Spring Symposium: AI and Health Communication.

Kim, E.-H. \& Kim, Y., 2010. Digital divide: Use of electronic personal health record by different population groups. In 32nd Annual International Conference of the IEEE Engineering in Medicine and Biology Society. Buenos Aires, Argentina, pp. 1759-62.

Lázaro, J.P. et al., 2010. Ambient assisted nutritional advisor for elderly people living at home. In 32nd Annual International Conference of the IEEE EMBS. Buenos Aires, Argentina, pp. 198-203.

Lexis, M. et al., 2013. Activity monitoring technology to support homecare delivery to frail and psychogeriatric elderly persons living at home alone. Technology and Disability, 25, pp.189-197.

Megalingam, R.K. et al., 2012. Elder health care: Blood Pressure measurement. 2012 Annual IEEE India Conference (INDICON), pp.747-752.

Misselhorn, C., Pompe, U. \& Stapelton, M., 2013. Ethical considerations regarding the use of social robots in the fourth age. The Journal of Gerontopsychology and Geriatric Psychiatry, 26(2), pp.121-133.

Mortenson, W. et al., 2013. Effects of an Assistive Technology Intervention on Older Adults with Disabilities and Their Informal Caregivers. American Journal of Physical Medicine \& Rehabilitation, 92(4), pp.297-306.

Norman, D.A. \& Draper, S.W., 1986. User Centered System Design; New Perspectives on Human-Computer Interaction, Hillsdale, NJ.: Lawrence Erlbaum.

Onyimadu, O., Harding, F. \& Briggs, J., 2011. Designing a Telecare Product for the Elderly. In Proceedings of the 5th International ICST Conference on Pervasive Computing Technologies for Healthcare. Ieee, pp. 336-339.

Parks, J., 2010. Lifting the burden of women's care work: Should robots replace the "human touch"'? Hypatia, 25(1), pp.100-120.

Piau, a et al., 2014. Aging society and gerontechnology: a solution for an independent living? The journal of nutrition, health \& aging, 18(1), pp.97-112. 
Rashidi, P. \& Mihailidis, A., 2013. A survey on ambient-assisted living tools for older adults. IEEE journal of biomedical and health informatics, 17(3), pp.579-90.

Rodríguez, S. et al., 2014. Multi-Agent Information Fusion System to manage data from a WSN in a residential home. Information Fusion, (2014).

Rokach, L. \& Maimon, O., 2008. Data mining with decision trees: theory and applications, World Scientific Pub Co Inc.

Rosenberg, E., Jullamate, P. \& Azeredo, Z., 2009. Informal caregiving: cross-cultural applicability of the person-environment model. Health Sociol, 18, pp.399-411.

Shankar, K.N. et al., 2014. Burden in caregivers of cognitively impaired elderly adults at time of hospitalization: a cross-sectional analysis. Journal of the American Geriatrics Society, 62(2), pp.27684.

Shin, J.H. et al., 2011. Detection of Abnormal Living Patterns for Elderly Living Alone Using Support Vector Data Description. IEEE Transactions on Information Technology in Biomedicine, 15(3), pp.438-448.

Silva, A.L. et al., 2013. The needs of informal caregivers of elderly people living at home: an integrative review. Scandinavian journal of caring sciences, 27(4), pp.792-803.

Skiba, D.J., 2014. The Connected Age: Big Data \& Data Visualization. Nursing Education Perspectives, 35(4), pp.267-269.

Sorell, T. \& Draper, H., 2014. Robot carers, ethics, and older people. Ethics and Information Technology, 16(3), pp.183-195.

Soto-Mendoza, V. \& Garcia-Macias, J.A., 2014. Lessons Learned from a Long-Running Assistive System for Geriatric Care. In L. Pecchia et al., eds. Ambient Assisted Living and Daily Activities. Belfast, Ireland: Springer, p. 83.

Sparrow, R. \& Sparrow, L., 2006. In the hands of machines? The future of aged care. Minds and Machines, 16(2), pp.141-161.

Su, C. \& Yude, W., 2012. The Care Of The Elderly With Chronic Disease Based On Electronic Health Records. In 2012 International Symposium on Information Technology in Medicine and Education. pp. 760-763.

Sun, H. et al., 2010. The missing ones: Key ingredients towards effective ambient assisted living systems. Journal of Ambient Intelligence and Smart Environments, 2(2).

Suryadevara, N.K., Quazi, M.T. \& Mukhopadhyay, S.C., 2012. Intelligent Sensing Systems for Measuring Wellness Indices of the Daily Activities for the Elderly. In 2012 Eighth International Conference on Intelligent Environments. Ieee, pp. 347-350.

UNFPA, 2012. State of the world population. Available at: http://www.unfpa.org/swp [Accessed January 7, 2013].

Vallor, S., 2011. Carebots and caregivers: Sustaining the ethical ideal of care in the 21st century. Philosophy and Technology, 24, p.254.

Wald, A., 2004. Sequential Analysis, Courier Dover Publications.

World Health Organization, 2012. Ageing. Available at: http://www.who.int/ [Accessed July 2, 2013].

Yedidia, M. \& Tiedemann, A., 2008. How do family caregivers describe their needs for professional help? $J$ Social Work Educ, 44, pp.43-47.

Zavala-Ibarra, I. \& Favela, J., 2012. Ambient videogames for health monitoring in older adults. In 2012 Eighth International Conference on Intelligent Environments. Ieee, pp. 27-33.

Zhang, D. et al., 2012. Social and Community Intelligence: Technologies and Trends. IEEE Software, 29, pp.88-92.

\#

Received XXX YYYY; revised XXX YYY; accepted XXXX YYYY 